\title{
A rainfall disaggregation scheme for sub-hourly time scales: Coupling a Bartlett-Lewis based model with adjusting procedures
}

\author{
Panagiotis Kossieris ${ }^{\mathrm{a},}{ }^{,}$, Christos Makropoulosa, Christian Onof ${ }^{\mathrm{b}}$ and Demetris \\ Koutsoyiannis ${ }^{\mathrm{a}}$ \\ aDepartment of Water Resources, Faculty of Civil Engineering, National Technical University of Athens, \\ Heroon Polytechneiou 5, GR-157 80 Zographou, Greece \\ ${ }^{b}$ Department of Civil and Environmental Engineering, Imperial College, London, UK \\ * Corresponding author, Tel. ++302107722886; e-mail: pkossier@central.ntua.gr
}

Many hydrological applications, such as flood studies, require the use of long rainfall data at fine time scales varying from daily down to 1 minute time step. However, in the real world there is limited availability of data at sub-hourly scales. To cope with this issue, stochastic disaggregation techniques are typically employed to produce possible, statistically consistent, rainfall events that aggregate up to the field data collected at coarser scales. A methodology for the stochastic disaggregation of rainfall at fine time scales was recently introduced, combining the Bartlett-Lewis process to generate rainfall events along with adjusting procedures to modify the lower-level variables (i.e., hourly) so as to be consistent with the higher-level one (i.e., daily). In the present paper, we extend the aforementioned scheme, initially designed and tested for the disaggregation of daily rainfall into hourly depths, for any sub-hourly time scale. In addition, we take advantage of the recent developments in Poisson-cluster processes incorporating in the methodology a 
Bartlett-Lewis model variant that introduces dependence between cell intensity and duration in order to capture the variability of rainfall at sub-hourly time scales. The disaggregation scheme is implemented in an R package, named HyetosMinute, to support disaggregation from daily down to 1-minute time scale. The applicability of the methodology was assessed on a 5-minute rainfall records collected in Bochum, Germany, comparing the performance of the above mentioned model variant against the original Bartlett-Lewis process (non-random with 5 parameters). The analysis shows that the disaggregation process reproduces adequately the most important statistical characteristics of rainfall at wide range of time scales, while the introduction of the model with dependent intensity-duration results in a better performance in terms of skewness, rainfall extremes and dry proportions.

Keywords: rainfall stochastic simulation; rainfall disaggregation; Poisson-cluster models; Bartlett-Lewis models; adjusting procedures; fine time-scales 


\section{Introduction}

Stochastic disaggregation is aimed at generating synthetic rainfall series which are fully consistent with the given data at a coarser scale and, at the same time, statistically consistent with the lower-level process. This approach enables the preservation of the stochastic structure of the process at multiple time scales, which could not be ensured via the inverse procedure, i.e., aggregation of the lower-level variables to derive the process at a coarser level (Koutsoyiannis, 2003a, 2001).

Disaggregation is of high importance especially in the study of rainfall at fine time scales (i.e., sub-monthly) which is the temporal resolution of interest for challenging hydrological applications such as the simulation of the hydrologic response of urban catchments in flood modelling applications, the design and implementation of real time control systems or the design and operation of combined sewer overflows (Bennett et al., 2013; Cowpertwait et al., 2006; Hingray et al., 2002; Koutsoyiannis and Foufoula-Georgiou, 1993; Koutsoyiannis, 1994; Segond et al., 2007). In this context, disaggregation can be employed to enrich the, frequently limited or even unavailable, fine-scale measurements (e.g. hourly and sub-hourly) with possible realizations of rainfall events, taking advantage of the longer rainfall records that do exist at coarse time scales (e.g. daily and above).

During the last decade, the problem of single-site disaggregation of rainfall at fine time scales has been studied systematically with the use of Poisson-cluster processes. The models of this category represent the physical precipitation mechanism through clustered rectangular pulses that occur in continuous time according to the Poisson point process. Clustering provides the flexibility of representing the complex phenomenon of rainfall at different time scales through a simplified and parsimonious approach. Rodriguez-Iturbe et 
al. (1987) first introduced and studied two alternative types of clustering, the BartlettLewis process and Neyman-Scott process, after observing the inability of simple Poisson models to reproduce the statistical characteristics of rainfall at multiple time scales (Rodriguez-Iturbe et al., 1987, 1984). In general, the two models have the same structure, while their main difference lying in the way that pulse origins are distributed within a cluster. Since then, various extensions and modifications of the original models have been proposed aiming to remedy weaknesses in reproducing the proportion of dry intervals and the distribution of extremes (Entekhabi et al., 1989; Gyasi-Agyei and Willgoose, 1997; Kim et al., 2013a; Onof and Wheater, 1994a; Rodriguez-Iturbe et al., 1988). The literature reports numerous empirical investigations that examine the performance of the Poissoncluster models in a wide range of rainfall types and climatic conditions. The models have been successfully fitted to data of various fine time scales from England (Cameron et al., 2000; Cowpertwait, 1991; Entekhabi et al., 1989; Onof and Wheater, 1994a, 1994b, 1993), Scotland (Glasbey et al., 1995), Belgium (Verhoest et al., 1997), Switzerland (Paschalis et al., 2014), Germany (Kaczmarska et al., 2014), Spain (Khaliq and Cunnane, 1996), Ireland (Khaliq and Cunnane, 1996), South Africa (Smithers et al., 2002), New Zealand (Cowpertwait et al., 2007), Australia (Gyasi-Agyei and Willgoose, 1997; Gyasi-Agyei, 1999; Wasko et al., 2015), Greece (Derzekos et al., 2005; Kossieris et al., 2015, 2013), Italy (Bo et al., 1994; Islam et al., 1990), United States (Bo et al., 1994; Kim et al., 2016, 2013b; Rodriguez-Iturbe et al., 1988, 1987; Velghe et al., 1994) and Korean Peninsula (Kim et al., 2014). Further to the single-site case, Poisson-cluster models have been also developed for the simulation of rainfall in space and time (e.g. Burton et al., 2008; Cowpertwait, 2010, 2002; Cox and Isham, 1988; Paschalis et al., 2013). Comprehensive reviews of the recent 
developments on Poisson-cluster models have been conducted by Onof et al. (2000) and Wheater et al. (2005), while more details are provided in section 2.1.

The aggregation and disaggregation properties of the Bartlett-Lewis model were first examined by Bo et al. (1994) who showed that the model can adequately reproduce the main statistics of hourly up to 12-hour time scale when 24- and 48-hour statistics are used to estimate the model parameters. On the contrary, a similar analysis for the Neyman-Scott model (Cowpertwait et al., 1996a) showed that the variability of hourly rainfall is not sufficiently reproduced when model fitting is based only on daily data. To improve model performance, the authors examine the use of neighbouring data to establish an empirical relationship between hourly and daily variance, and then the estimated variance is used to fit the model.

Despite the fact that the researchers mentioned above have studied the problem of simulating rainfall at fine time scales using only coarser-resolution data, their work cannot be considered "disaggregation" in the strict sense of the term, given that they do not establish consistency between higher- and lower-level variables. This problem was first examined by Glasbey et al. (1995) who employed the Bartlett-Lewis model to generate a large record of hourly and daily data (e.g. 1000 years). For each given sequence of wet days (the authors assumed as independent a sequence of wet days that is preceded and followed by at least one dry day), the algorithm searches in the record for a sequence of the same length that is in close agreement with the original one. For the chosen sequence, the synthetic hourly depths are rescaled to add up exactly to given daily values. However, this rescaling procedure causes an overestimation of the variance of hourly intensities. To remedy this weakness, the authors examined another ad-hoc model that searches for best 
matching sequence on the basis of daily totals of 3 days. Despite the improvement in the statistics achieved, this method does not have the general character of the initial scheme, since a 3-day period cannot necessarily be considered as independent (Glasbey et al., 1995; Koutsoyiannis and Onof, 2001).

More recently, Koutsoyiannis and Onof (2001) extended the general coupling methodology of Koutsoyiannis (2001) to disaggregate daily rainfall into hourly depths. Their scheme combines the Bartlett-Lewis model for the generation of synthetic rainfall depths along with an adjusting procedure (Koutsoyiannis and Manetas, 1996) to establish consistency with the given daily depths. Specifically, for a cluster of wet days, the rainfall model runs several times and the generated sequence that best matches the original one in terms of daily totals, is chosen. Then, the synthetic sequence of hourly rainfall depths is modified according to a proportional adjusting procedure to add up to the given daily depths. The methodology was implemented in the computer program Hyetos (Koutsoyiannis and Onof, 2000). As the authors pointed out, this method shares some similarities to the earlier disaggregation scheme of Glasbey et al. (1995), but its structure is different. Firstly, it is applied directly to each cluster of wet days without requiring the $a$ priori construction of an auxiliary large series of daily data. Further to that, the model incorporates distinct levels of repetitions for the generation of temporal characteristics of sequences and the intensities of pulses, reducing significantly the required computational time. Successful application of Koutsoyiannis and Onof's disaggregation scheme (2001) has been reported from Abdellatif et al. (2013), Debele et al. (2007), Engida and Esteves (2011), Pui et al. (2012) and Segond et al. (2007, 2006). The single-site model was generalized for the multivariate case by Koutsoyiannis et al. (2003), while the single-site 
Hyetos model has been employed into different hybrid schemes for the spatio-temporal disaggregation of daily rainfall (Gyasi-Agyei and Mahbub, 2007; Gyasi-Agyei and Willgoose, 1997; Gyasi-agyei, 2005; Gyasi-Agyei, 1999; Segond et al., 2006) .

To date, the applications of the Poisson-cluster models in a disaggregation framework concern mainly the generation of consistent hourly rainfall depths from given daily values. Regarding the sub-hourly time scales, the literature is more restricted and concerns mainly ad hoc techniques rather generic methodologies. Cowpertwait et al. (1996b) developed a process for the single-site disaggregation of hourly rainfall depths into smaller time intervals using the Neyman-Scott rectangular pulse model. Some shortcomings of this model in reproducing the temporal structure and the extreme behaviour of the disaggregated 5-minute rainfall depths were noticed by Onof et al. (2005). Gyasi-Agyei and Mahbub (2007) extended the multi-site regional hybrid disaggregation model of GyasiAgyei (2005) for sub-hourly time scales. It is also worth mentioning the hybrid scheme of Anis and Rode (2015) that uses the Hyetos software to generate hourly rainfall depths and then a micro-canonical cascade model (Onof et al., 2005; Sivakumar and Sharma, 2008) to disaggregate the hourly depths into 10-minute data. A similar approach was followed earlier by Laloy and Bielders (2009) who applied a symmetrical double-triangular hydrograph method to hourly data from Hyetos in order to generate 1-minute consistent rainfall depths.

Recently, special focus has been given in the extension of the Poisson-cluster models to capture rainfall characteristics at sub-hourly time scales. The modifications concern mainly either the incorporation of a third level of process of instantaneous pulses to enhance model flexibility in reproducing different storm profiles (Cowpertwait et al., 2007; 
Cowpertwait, 2004) or the introduction of a dependence between rainfall intensity and cell duration (Kaczmarska et al., 2014). A more comprehensive description of these models is given in section 2.1.

In this paper we build upon the coupling disaggregation methodology of Koutsoyiannis and Onof (2001) and examine its extension and applicability to sub-hourly time scales. The new model extends the original one in two important ways:

1. The disaggregation scheme, initially designed and tested for the disaggregation of daily rainfall into hourly depths, is now extended and verified for any sub-hourly time scale.

2. The recently developed Bartlett-Lewis model that assumes dependence between cell intensity and duration (Kaczmarska et al., 2014) is introduced in the scheme to enable the adequate reproduction of the high variability that rainfall exhibits at super-fine time scales.

As a test case, we examined the performance of the methodology in the disaggregation of daily rainfall depths into 5-minute data, using a dataset from Bochum (Germany). The new aforementioned variant is compared against the original BartlettLewis model (Rodriguez-Iturbe et al., 1987) allowing us to reach conclusions on the performance of these models within a disaggregation framework. In our test case, we take advantage of the fact that 5-minute field data are actually available and hence can be used both for model fitting and validation. Subsequently, our application of the disaggregation model addresses the issue of enhancing daily data records when observed data at finer time scales are available at that gauge or at neighbouring gauges. The results of model application are presented in section 4 . 
Our primary motivation for this study arises from the need for a generic and parsimonious, as well as operational, model that is easily applicable for the simulation of rainfall at any fine time scale. The model was implemented in a computer program developed by the authors for this purpose, named HyetosMinute (see section 3), that allows both the sequential simulation and the disaggregation of rainfall at any scale from daily down to 1-minute scale. 


\section{Methodology and tools}

A general framework for the generation of synthetic series that is fully consistent with given data of a coarser timescale and, at the same time, statistically consistent with the process under study, was initially studied by Koutsoyiannis (1994) and Koutsoyiannis and Manetas (1996) and later generalized by Koutsoyiannis (2001). Unlike most disaggregation techniques that involve simultaneously both models of different time scales in one mathematical expression (Grygier and Stedinger, 1988; Stedinger and Vogel, 1984; Stedinger et al., 1985; Valencia R. and Schaake L., 1972; Valencia and Schaake, 1973), this scheme is based on the coupling of independent stochastic models, each applied separately to simulate the process at a specific time scale, through mathematical transformations. The coupling transformation (also termed 'adjusting procedure') modifies the lower-level variables in order to establish consistency with the higher-level time series, ensuring at the same time the exact preservation of certain statistics (i.e., marginal and joint second-order statistics) or even the complete distribution of the former. To improve the approximation of the statistics which are not explicitly preserved by the adjusting procedure (i.e., skewness coefficient), the methodology employs an iterative sampling procedure. Specifically, the lower-level model runs several times and the generated auxiliary series that is in closest agreement with that of the higher-level is chosen for adjusting (Koutsoyiannis and Manetas, 1996).

The above coupling framework, combined with the appropriate stochastic models (in their univariate or multivariate variant), has been successfully applied to reproduce the statistical behaviour and characteristic properties of hydrologic processes at a wide range of time scales (Efstratiadis et al., 2014; Koutsoyiannis and Manetas, 1996; Koutsoyiannis 
and Onof, 2001; Koutsoyiannis, 2001; Koutsoyiannis et al., 2003). To disaggregate annual rainfall depths into monthly amounts, Koutsoyiannis (2001) coupled, through a linear transformation, a symmetric moving average model with long-term memory autocorrelation function (Koutsoyiannis, 2000), for the reproduction of Hurst-Kolmogorov dynamics (Koutsoyiannis, 2011, 2003b) at annual scale, with a periodic autoregressive gamma model, i.e. a PAR(1) model, for the simulation of monthly seasonality. For the disaggregation into daily amounts, a proportional adjusting procedure is applied to daily series which is generated by another PAR(1) model combined with ad hoc truncation techniques for the reproduction of the intermittent nature of daily process (Efstratiadis et al., 2014; Koutsoyiannis, 2001). The three-level stochastic disaggregation framework has been successfully applied in several hydrological applications (e.g., Efstratiadis et al., 2015; Nalbantis et al., 2011; Tsekouras and Koutsoyiannis, 2014; Tsoukalas and Makropoulos, 2015; Tsoukalas et al., 2016).

As indicated above, this coupling methodology was extended for the univariate (Koutsoyiannis and Onof, 2001) as well as multivariate (Koutsoyiannis et al., 2003) disaggregation of daily rainfall into hourly steps. In the univariate case, the Bartlett-Lewis rectangular pulse model is employed to generate hourly synthetic series. Then, the proportional adjusting procedure is applied to modify the hourly depths so as to be consistent with the given daily data. In the multivariate case, the generation of the lowerlevel variables is conducted through a multivariate gamma autoregressive AR(1) model, combined with truncation techniques for the reproduction of dry intervals, while the generated hourly depths become consistent with the daily values via the general linear transformation. 
Here, we focus on the extension of the univariate disaggregation methodology into sub-hourly time scales, taking advantage of the recently developed variants of the BartlettLewis process, oriented to capture the high variability that precipitation exhibits at superfine time scales. Next, we provide an overview of the Bartlett-Lewis process as the rainfall model used in the present work (section 2.1) and then we describe in detail the disaggregation methodology (section 2.2).

\subsection{The Bartlett-Lewis rectangular pulse models}

As discussed in section 1, the Bartlett-Lewis rectangular pulse model belongs to the general category of Poisson-cluster models that simulate rainfall events via clusters of rectangular pulses that occur in continuous time. The ability of this type of model to reproduce the characteristics of rainfall at multiple time scales, even in cases where some of these time scales are not preserved explicitly by a fitting procedure (Bo et al., 1994), makes the model appropriate for disaggregation frameworks in which different time scales are involved (Gyasi-Agyei, 1999).

The basic assumptions of the Bartlett-Lewis clustering mechanism are (Fig. 1; Rodriguez-Iturbe et al., 1987):

- Storm origins $t_{i}$ occur in a Poisson process with rate $\lambda$ and each storm $i$ is associated with a random number of cells.

- Within each storm $i$, the origin $t_{i j}$ of each cell $j$ occurs following a second Poisson process with rate $\beta$, whereas the origin of first cell coincides with the storm origin. The time intervals of successive storm and cell origins are independent and identically distributed random variables that follow an exponential distribution. 
- Within each storm, the generation of cells terminates after a time span $v_{i}$ following the exponential distribution with rate $\gamma$. This implies that the number of cells per storm has a geometric distribution of mean $\mu_{c}=1+\beta / \gamma$.

- Each cell has a duration $w_{i j}$ following the exponential distribution with rate $\eta$.

- Each cell has an intensity $x_{i j}$ with a specific distribution. In the simplest version of the model, the exponential distribution with mean $\mu_{X}$ is assumed.

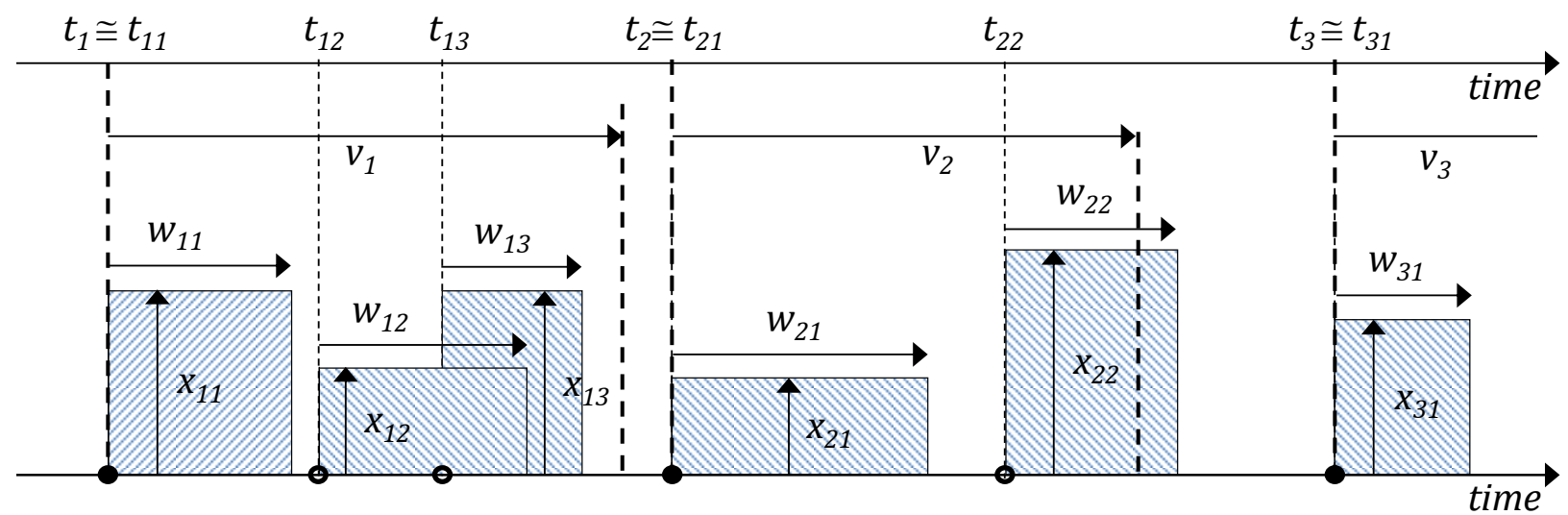

Fig. 1. Schematic representation of the Bartlett-Lewis clustering mechanism. Filled circles denote storm origins while open circles denotes cell arrivals.

Subsequently, the initially proposed model, hereinafter referred to as the Rectangular Pulse Bartlett-Lewis (RPBL) model, has 5 parameters: $\left\{\lambda, \beta, \gamma, \eta, \mu_{X}\right\}$. The model allows both for storm and cell overlapping, while the total rainfall intensity, $Y(t)$, at every instant $t$, is obtained by summing all active pulses at time $t$.

Despite the successful reproduction of the basic statistics (first- and second-order moments of rainfall depths) from hourly up to daily scale, the RPBL model showed some difficulties in reproducing the temporal characteristics of rainfall as expressed via the overestimation of the proportion of dry periods (Onof and Wheater, 1994b; RodriguezIturbe et al., 1988; Rodríguez-Iturbe et al., 1987; Velghe et al., 1994). The discrepancies are 
attributed to the fact that parameters $\gamma, \beta$ and $\eta$ which control the temporal properties of the process remain constant between different storms and subsequently the model is not able to reproduce different types of rainfall as well as the variability within storm events. Extended analysis on the temporal properties of dry periods and storm events of the Bartlett-Lewis clustering process has been conducted by Onof and Wheater (1994b, 1993; 1994a) and Onof et al. (1994).

To enhance the model's flexibility in generating a greater diversity of rainfalls, Rodriguez-Iturbe et al. (1988) modified the original model so that parameter $\eta$ is randomly varied from storm to storm according to the gamma distribution with a shape parameter $\alpha$ and a rate parameter $v$. The parameterization of the Random Parameter Bartlett-Lewis (BLRPR) model entails also that the cell origin rate $\beta$ and the storm duration rate $\gamma$ are also varied so that the ratios $\kappa:=\beta / \eta$ and $\varphi:=\gamma / \eta$ are kept constant. Subsequently, in BLRPR model, parameters $\beta$ and $\gamma$ are also random variables following gamma distribution with common shape parameter $\alpha$ and rate parameters $v / \kappa$ and $v / \varphi$, accordingly. The BLRPR model has 6 parameters: $\left\{\lambda, \alpha, v, \kappa, \varphi, \mu_{X}\right\}$.

In its simplest version, the model assumes the exponential distribution for cell intensity. However, some discrepancies in the distribution of extreme values at different time scales, especially for lower time scales and high return periods, have been observed (Onof and Wheater, 1994b, 1993; Rodriguez-Iturbe et al., 1988, 1987; Velghe et al., 1994; Verhoest et al., 1997). The performance of the model can be improved by incorporating a longer-tailed distribution for cell intensity. The gamma distribution has been examined (Onof and Wheater, 1994a) and implemented in some cases (Verhoest et al., 1997, 2010), while Weibull and Pareto distributions are also potential candidates. Alternatively, the use 
of third or higher order moments of rainfall depth in fitting procedure may result in the improvement of the extreme-values performance of the models (Cowpertwait, 1998; Verhoest et al., 2010; Wheater et al., 2005). Kim et al. (2013a) showed that the distribution of the maximum rainfall depths can be better reproduced by incorporating the inter-annual variability of monthly statistics in the estimation of the model parameters.

The Bartlett-Lewis model has been further extended to allow the reproduction of high variability of rainfall profile at sub-hourly time scales. In this framework, Cowpertwait et al. (2007) modified the RPBL model so that the constant rectangular cell intensities are replaced by a cluster of instantaneous pulses that occur following a third Poisson process of rate $\xi$. Subsequently, the BLIP model incorporates 3 Poisson processes that control respectively, the arrival of storms with rate $\lambda$, the arrival of cells within storms with rate $\beta$ and the arrivals of instantaneous pulses within cells with rate $\xi$. This model was further extended by Kaczmarska et al. (2014) in a way that cell duration parameter $\eta$ is randomly varied between storms following the BLRPR model. The BLIPR model implies that the ratio, $\omega:=\xi / \eta$, of instantaneous pulse arrival rate to the cell duration parameter is kept constant in the process, giving a total of 7 parameters: $\left\{\lambda, \alpha, v, \kappa, \varphi, \omega, \mu_{X}\right\}$.

Greater variability in temporal storm characteristics can be also achieved by superposing multiple independent processes for different types of rainfall (Cowpertwait et al., 2007; Cowpertwait, 2004). Cowpertwait et al. (2007) examined a superposed model consisting of two independent BLIP processes in order to simulate rainfall from 5-minute up to daily scale at a site in Kelburn (New Zealand). Despite the good performance of the model, the large number of independent parameters poses extra difficulties in the fitting procedure. Kaczmarska et al. (2014) examined further Cowpertwait et al. (2007)'s model 
along with a superposed model consisting of two RPBL model, in a 5-minute rainfall time series from a site in Bochum (Germany), showing that for both superposed models it was not possible to obtain a realistic and stable set of parameters.

As an alternative to the above discussed approaches, Kaczmarska et al. (2014) reparameterize the BLRPR model so as to introduce dependence between cell intensity and duration. Specifically, the cell intensity parameter $\mu_{X}$ varies between storms (similarly to $\eta$, $\beta$ and $\gamma$ ) so that the ratio $i:=\mu_{X} / \eta$ remains constant. Subsequently, the new model, hereinafter referred to as the BLRPRX model, has 6 parameters: $\{\lambda, \alpha, v, \kappa, \varphi, i\}$. The analysis showed that this model outperforms both the superposed models and those with a third layer of pulses in preserving the statistical characteristics of Bochum rainfall from 5minute up to daily.

\subsection{The disaggregation scheme}

In the disaggregation scheme, the synthetic rainfall depths that have been generated via one of the above-described Bartlett-Lewis models are modified so as to be consistent with the given daily series. In more detail, the methodology is formed as follows: Provided that a daily series $Z_{i}(i=1,2, \ldots)$ is known, a lower-level auxiliary series $\tilde{X}_{s}(s=1, \ldots, k)$ is generated via one of the above described Bartlett-Lewis models. Given that the auxiliary series has been produced independently to the higher-level variables, $\tilde{X}_{s}$ do not sum up to $Z_{i}$, but to some other quantities $\tilde{Z}_{i}$. The error in the additive property, i.e., the departure of $\tilde{Z}_{i}$ quantities from the corresponding higher-level variables $Z_{i}$, is allocated to the lowerlevel time series, through an adjusting procedure, and thus a modified series $X_{s}(s=$ $1, \ldots, k)$ is obtained. Now, the latter satisfies the additive property $\sum_{s=1}^{k} X_{s}=Z_{i}(i=1,2, \ldots)$. 
The special characteristics of rainfall series at fine time scales, i.e., large proportion of zero values, strong autocorrelation structure and highly-skewed distribution, establish the proportional adjusting procedure (Eq. (2)) as more appropriate to modify the auxiliary synthetic series (Koutsoyiannis and Onof, 2001). This procedure does not transform zero values to negative, as linear procedures do, and explicitly preserves the complete distribution for independent variables that have the gamma distribution with common scale parameter (Koutsoyiannis and Manetas, 1996). Further to that, it enables preservation of the full distribution for independent gamma distributed variables and provides a good approximation for dependent variables with gamma distribution (Koutsoyiannis, 1994). However, at fine time scales, such as hourly and even more so at sub-hourly scales, the autocorrelation is high and, hence, the assumption of independence among successive variables is not valid.

To improve the approximation of the statistics which are not explicitly preserved by the proportional adjusting procedure, an iterative process is employed. Specifically, the Bartlett-Lewis model runs several times and the sequence that is in closest agreement with the higher-level sequence (i.e., by means that a distance measure (Eq. (1)) is lower than an accepted limit $d_{a}$ ) is chosen to be transformed. To investigate the efficiency of this repetition scheme along with the proportional adjusting procedure, Koutsoyiannis and Onof (2001) conducted a series of "toy" disaggregation experiments, using a hourly synthetic series, generated via an intermittent gamma autoregressive process. The analysis showed that the adjusting procedure, irrespective of the value of $d_{a}$, does not create any bias in variation and skewness as long as the probability dry of each specified period is explicitly preserved by the lower-level model (i.e., the number of zero values of the 
generated series is kept constant and equal to that of the original sequence during repetitions). On the contrary, when the number of zero values is not known, a considerable increase in variation and skewness is observed. Despite the fact that this bias decreases by adopting low values for $d_{a}$, it can not be truly eliminated by increasing the number of repetitions. As a possible solution to this, a negative bias can be introduced to variation and skewness before model fitting (Koutsoyiannis, 2001). Regarding the approximation of autocorrelation, the bias is decreasing with the combined use of adjusting procedure and repetitions, for low limit $d_{a}$.

From a technical point of view, the application of the repetition scheme directly in a long simulation period may lead to extremely high computational times due to the difficulty in finding a sequence that matches the higher-level time series, by means of a distance measure. To cope with this issue, different sequences (clusters) of wet days, preceded and followed by at least one dry day, can be assumed stochastically independent, hence treated as such. This is in full compliance with the Bartlett-Lewis mechanism that entails that the time intervals between successive storms are independent variables with exponential distribution and common rate parameter. On the other hand, we can not consider and treat as independent successive sequences of wet intervals at sub-daily time scales. Due to this, the disaggregation into sub-hourly intervals is conducted always with reference to the known daily values and not to those of finer scales.

The high computational time may remain an issue for very long clusters of wet days. Due to this, the disaggregation scheme allows the random sub-division of very long sequences into sub-sequences in cases when the departure of the sum of generated lowerlevel variables from the given daily depths remains higher than an acceptable limit after a 
number of repetitions. In some cases, this process may lead to nested, successive subdivisions of the initially given cluster into many sub-clusters.

Aiming to further reduce the computational time, two different levels of repetitions is implemented, taking advantage of the fact that in the Bartlett-Lewis mechanism the temporal characteristics of storm events are generated independently of the cell intensities. Subsequently, for each cluster of wet days, the Bartlett-Lewis model runs several times to establish, in the first phase, the appropriate wet/dry structure, and in a second phase the intensity profile of the event.

For a cluster of $L$ wet days, the disaggregation of daily rainfall into any sub-hourly depths (e.g., 5-minute) comprises the following steps (adapted from Koutsoyiannis and Onof (2001), see Fig. 2):

Step 1: The Bartlett-Lewis model (i.e., RPBL, BLRPR or BLRPRx) generates sequences of storms and cells, at the specific sub-hourly time scale (e.g. 5-minute), until a cluster of exactly $L$ wet days, followed by one or more dry days, is obtained.

In the case that the cluster has been formed successfully within an allowed number of repetitions, $n_{0}$, the process continuous to Step 2. Otherwise, the cluster of $L$ wet days is subdivided randomly into sub-clusters with smaller lengths. In this case, the disaggregation process applied to each cluster independently, starting from the current Step 1.

Step 2: For the formed sequence of storms and cells, the cell intensities are generated and the synthetic daily depths are calculated. The synthetic daily depths are compared to the original ones according to the formula: 


$$
d=\left[\sum_{i=1}^{L} \ln ^{2}\left(\frac{Z_{i}+c}{\tilde{Z}_{i}+c}\right)\right]^{1 / 2}
$$

where $Z_{i}$ and $\tilde{Z}_{i}$ are the original and synthetic daily depth of day $i$ of the wet cluster, and $c$ (= $0.1 \mathrm{~mm}$ ) a small constant inserted to prevent domination by the very low depths. At the same time, the logarithm prevents the domination of very high values. If the departure $d$ is smaller than an acceptable limit $d_{a}$, the algorithm moves to Step 3. Otherwise, a new sequence of cell intensities is generated for the same arrangement of storm and cells. The generation of new cell intensities continues for an allowed number of repetitions, $n_{1}$. If, however, the model cannot establish an appropriate sequence of intensities the temporal arrangement of storms and cells is discarded and replaced by a new one, thus returning at Step 1.

This process holds until the total repetitions, i.e. repetitions for established wet/dry sequences and cell intensities, exceeds an allowed number, $n_{2}$. After this number, the initial sequence is subdivided randomly into shorter sub-sequences and the process begins from Step 1 for each new sub-sequence independently. In some cases, this process may lead to nested, successive subdivisions of the initially given cluster into many sub-clusters.

Step 3: For the chosen sequence, the rainfall depths of the lower level $X_{S}$ (e.g. 5minute) are obtained by modifying the generated depths $\tilde{X}_{s}$ according to the proportional adjusting procedure:

$$
X_{s}=\tilde{X}_{s}\left(\frac{\sum_{i=1}^{L} Z_{i}}{\sum_{i=1}^{L} \tilde{Z}_{i}}\right) \quad(s=1, \ldots, k)
$$

where $Z_{i}$ and $\tilde{Z}_{i}$ are the original and generated daily depth of day $i$ of the wet cluster and $k$ is the number of lower-level variables within one higher-level period. 


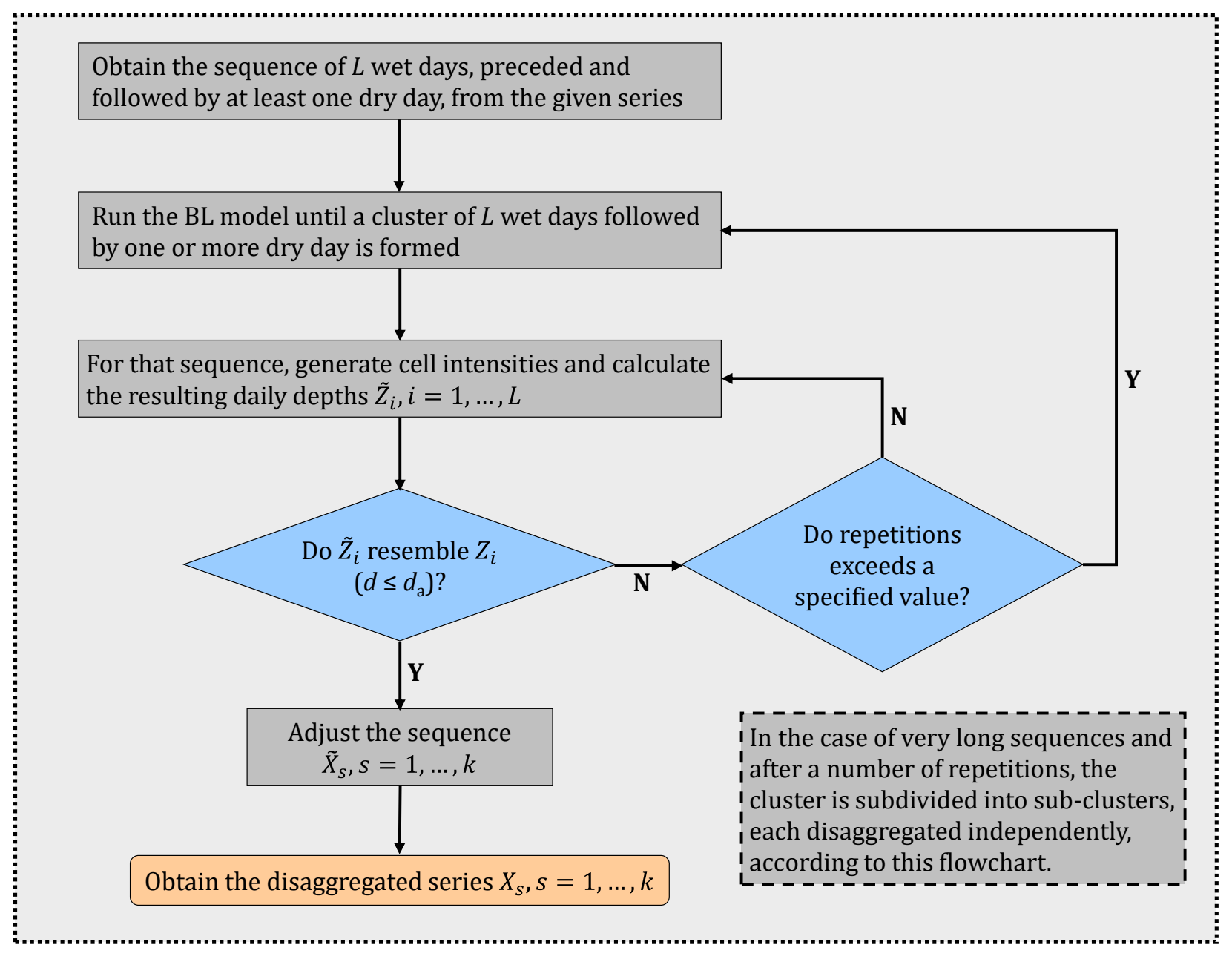

Fig. 2. Flow chart of the disaggregation scheme for a cluster of $L$ wet days (after adjustment from Koutsoyiannis and Onof (2001)) 


\section{Software description - "HyetosMinute"}

Within this work, a new software program, named HyetosMinute ${ }^{1}$, was created to allow for the temporal stochastic simulation of rainfall at fine time scales. The software was coded in the programming language R (R Core Team, 2014), with some parts of code implemented in C. HyetosMinute incorporates various new functionalities that extend the stand-alone Hyetos software (Koutsoyiannis and Onof, 2000) in various ways: (1) generation of synthetic rainfall data, either via sequential simulation or disaggregation, at sub-hourly time scales, and specifically down to 1-minute time scale, (2) implementation and incorporation of the recently developed Bartlett-Lewis model with randomized intensity parameter (Kaczmarska et al., 2014) in the disaggregation scheme, further to the original (Rodriguez-Iturbe et al., 1987) and the random parameter $\eta$ (Rodriguez-Iturbe et al., 1988) versions of the model, (3) implementation of an enhanced-version of the Evolutionary Annealing-Simplex $\left(\mathrm{EAS}^{2}\right)$ optimization algorithm for the estimation of model parameters.

Depending on data availability, the package operates either (i) in testing mode, in cases where historical rainfall depths are imported to enable comparisons (by means of various statistics), or (ii) in full operational mode if only daily data are available. For all modes, the generated rainfall series are delivered to the user either in $\mathrm{R}$ console or are exported in external files for further processing. The package also has several graphical capabilities that allow the direct representation and comparison of statistical characteristics of original and synthetic data (e.g. marginal statistics, conditional statistics,

${ }^{1}$ HyetosMinute is free and available online at: $\mathrm{http}: / /$ www.itia.ntua.gr/en/softinfo/3/

2 EAS and SEEAS (i.e., the surrogate-enhanced extension) are free and available online at: http://www.itia.ntua.gr/en/softinfo/29/ 
temporal properties of rainfall, autocorrelation structure etc.). Further to that, the hyetographs of storm events are also plotted and exported for further analysis.

The package also enables the estimation of the Bartlett-Lewis model parameters via an enhanced-version of the EAS optimization method (Kossieris et al., 2015), originally developed by Efstratiadis and Koutsoyiannis (2002; Tsoukalas et al., 2016). The method combines the strength of simulated annealing in locating regions of attraction in rough search spaces along with the efficiency of the downhill simplex method (Nelder and Mead, 1965 ) in smoother spaces. The search procedure is based on the evaluation of a population either via quasi-stochastic geometric transformations, inspired by the downhill simplex method, or fully-probabilistic transitions (mutations). The degree of randomness is determined by an adaptive annealing cooling schedule that reduces system temperature so as all transitions become more deterministic as search proceeds. The major difference to the original EAS (Efstratiadis and Koutsoyiannis, 2002) involves the reflection step through weighted centroid (proxy of the gradient of search space) instead of the geometric one. This modification accelerates the search, enabling the faster convergence to region of optimum. The platform allows the user to fully configure the multiple arguments of the fitting problem, i.e., statistics to be included in the objective function, form of the objective function, parameter bounds, population size, etc. This is a major advancement with respect to earlier versions of the methodology, which did not implement parameter estimation. 


\section{Case study}

The disaggregation methodology was tested on a 69-year time series of 5-min rainfall data, collected from a single site in Bochum (Germany). This extensive dataset has been used in the past by Kaczmarska et al. (2014) to assess the applicability of different BartlettLewis models in the modeling of rainfall from 5-minute up to the daily scale. In the present work, taking advantage of the good fitting achieved for a series of model variants, we applied the disaggregation methodology to generate 5-minute synthetic data that sum up exactly to the historical daily values. The analysis was conducted on a monthly basis, due to the seasonality that rainfall characteristics exhibit, and for multiple time scales, i.e., from 5minute up to daily.

In the present case study, two variants of the Bartlett-Lewis model, with exponentially distributed cell intensities, were used, i.e., the original, 5-parameter, RPBL model (Rodriguez-Iturbe et al., 1987) as well as the 6-parameter, BLRPRx, random model with randomized intensity parameter (Kaczmarska et al., 2014). The selection of these two models was based on a comparative analysis, on Bochum's data, which showed that the BLRPRx model outperforms the two instantaneous pulse models, i.e., BLIP and BLIPR models, intended for the reproduction of variability of sub-hourly rainfall (Kaczmarska et al., 2014). In addition, the same analysis revealed that the 6-parameter randomized BLRPR model achieves an equally good fitting with the RPBL model, and hence the most parsimonious model is selected for further analysis. The performance of the two models was assessed in terms of preserving the main statistical characteristics of observed data, both in the sequential simulation and disaggregation framework. 
The parameter sets used in the present study were based on the findings of Kaczmarska et al. (2014) who achieved a good fit of the RPBL and BLRPRx model onn the Bochum dataset, for a wide range of time scales and properties. Following the Wheater et al. (2005) approach, the afore-mentioned authors employed a two-step approach that handles model fitting as optimization problem where the objective is the minimization of the departure between the theoretical expressions of the moments and the corresponding observed values. Initially, a number of optimizations are performed via the downhill simplex algorithm (Nelder and Mead, 1965), using different starting values for the parameters. Then, a gradient-based method runs several times in order to refine further the optimum solution obtained from the first step (Chandler et al., 2010).

The optimization problem was formulated according to the generalized method of moments, with the number of properties inserted in the objective function exceeding the number of model parameters (Jesus and Chandler, 2011). In this framework, the properties included in the fitting procedure were the mean of hourly rainfall as well as the coefficient of variation, lag-1 auto-correlation, and skewness at time scales of 5-minutes, 1 hour, 6 hours and 24 hours. Despite the fact that the proportion dry could also be included in the fitting as an important property of the process, it is kept as validation criterion for assessing the models' performance.

All simulations were performed using HyetosMinute package. Regarding the parameters of the disaggregation scheme, a preliminary analysis showed that the optimum value for the maximum allowed distance $d_{a}$ is 0.1 and for the maximum number of total repetitions $n_{2}$ is 5000 . Despite the fact that smaller values for $d_{a}$ lead to better results for some statistics (e.g. skewness) and for some months, the computational time becomes 
extremely high without any noticeable improvement in the overall performance, i.e. different time scales and months. At the same time, higher values of $n_{2}$ parameter (e.g. 10000) do not result in bias reduction.

As it has already mentioned above, the evaluation of the disaggregation scheme as well as of the Bartlett-Lewis model suites was conducted on the basis of certain statistical properties of rainfall at 5-minute, 1-, 6- and 24-hour time scales. Graphical representations of the results are given in Fig. 3 through Fig. 11. Each graph displays a specific property for all months, deriving from: historical data, simulated data without disaggregation via the RPBL and BLRPRx model, and finally disaggregated data using the two models. Having the 5-minute rainfall depths generated by the model, the data at coarser temporal scales were obtained via aggregation. Given that the disaggregation scheme establishes full consistency between 5-min and daily scale, in the plots of the 24-hour statistics, the disaggregated data match the observed exactly.

The statistical properties that are explicitly preserved by the Bartlett-Lewis models, i.e., mean, variance, lag-1 correlation and skewness coefficient, are displayed in Fig. 3 to Fig. 6. Regarding the mean and variance of rainfall depth, a very good agreement between the historical and disaggregated series was achieved for all months and time scales. The small overestimation that is noticed in the variance of summer months is mainly attributed to the large proportion of zero values and the high skewness coefficients that data exhibit in these months (see section 2.2 for more details on possible sources of bias). In terms of the coefficient of skewness (Fig. 5), the disaggregation model follows in general the performance of the rainfall models. It is clear that both RPBL and BLRPRX models tend to underestimate the skewness, especially in summer months and at the 5-min time scale, 
while a better approximation is achieved with the BLRPRx model. As in the case of variance, the disaggregation process introduces a slight positive bias at the 5-min and 1-hour theoretical skewness of RPBL model, which in this case leads to a better approximation of historical values. As we can see this bias is significantly smaller than the departure between the historical and the theoretical values, and, hence, a better fit of the rainfall models regarding skewness coefficient can lead to the better performance of the disaggregation model. Regarding the lag-1 autocorrelation coefficient (Fig. 6), the rainfall models achieve a very good fitting to the historical values, while an underestimation is noticed at 24-hour time scale. As it is obvious, the disaggregation model with both rainfall models also preserves exactly the coefficients at 5-min and 1-hour scales, introducing a small negative bias in the values of the 6-hour scale.

Fig. 7 displays the proportion of dry intervals in the entire period. As previously discussed, this property has not been introduced in the fitting procedure of the rainfall models, and, hence, it is a validation criterion both for Bartlett-Lewis models and disaggregation scheme. We observe that, for all time scales and months, the BLRPRx model achieves a better approximation of the historical values compared to the RPBL model, while some higher discrepancies are noticed in summer months. In the disaggregation framework, the two rainfall models exhibit different behaviours. The repetitions schemes and adjusting procedures do not introduce any bias in the case of RPBL model, while in the case of BLRPRx model, the disaggregated series have higher values of proportion dry compared to the theoretical property of the model. However, in the disaggregation, the model BLRPRX remains superior to the original RPBL model. To further improve model 
performance, the proportion dry can be inserted in the fitting procedure, enabling the better preservation of this property. 

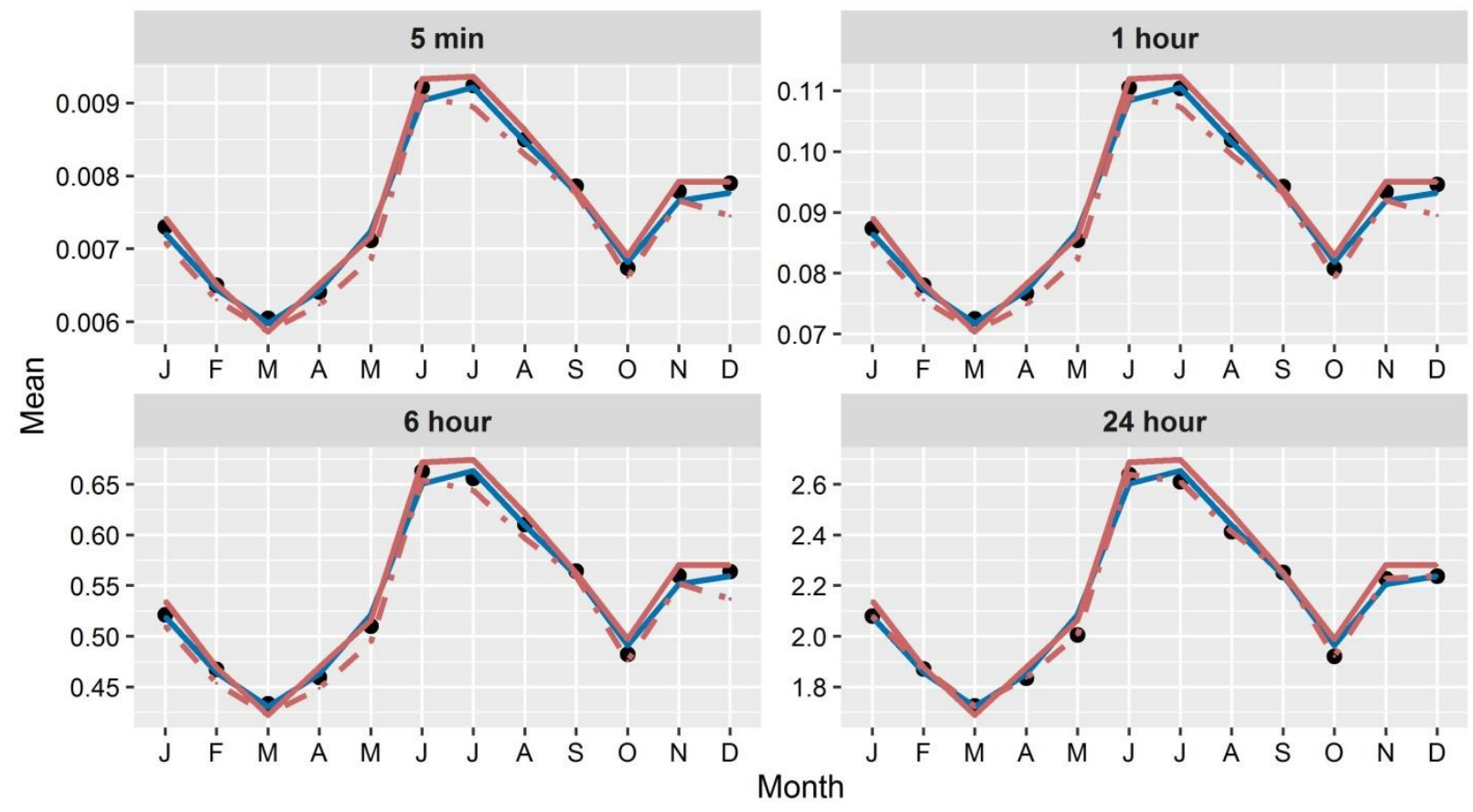

- Obs - BLRP . - BLRP-Disag - BLRPRX - - BLRPRX-Disag

Fig. 3. Comparison of mean of the historical and synthetic data, by month.

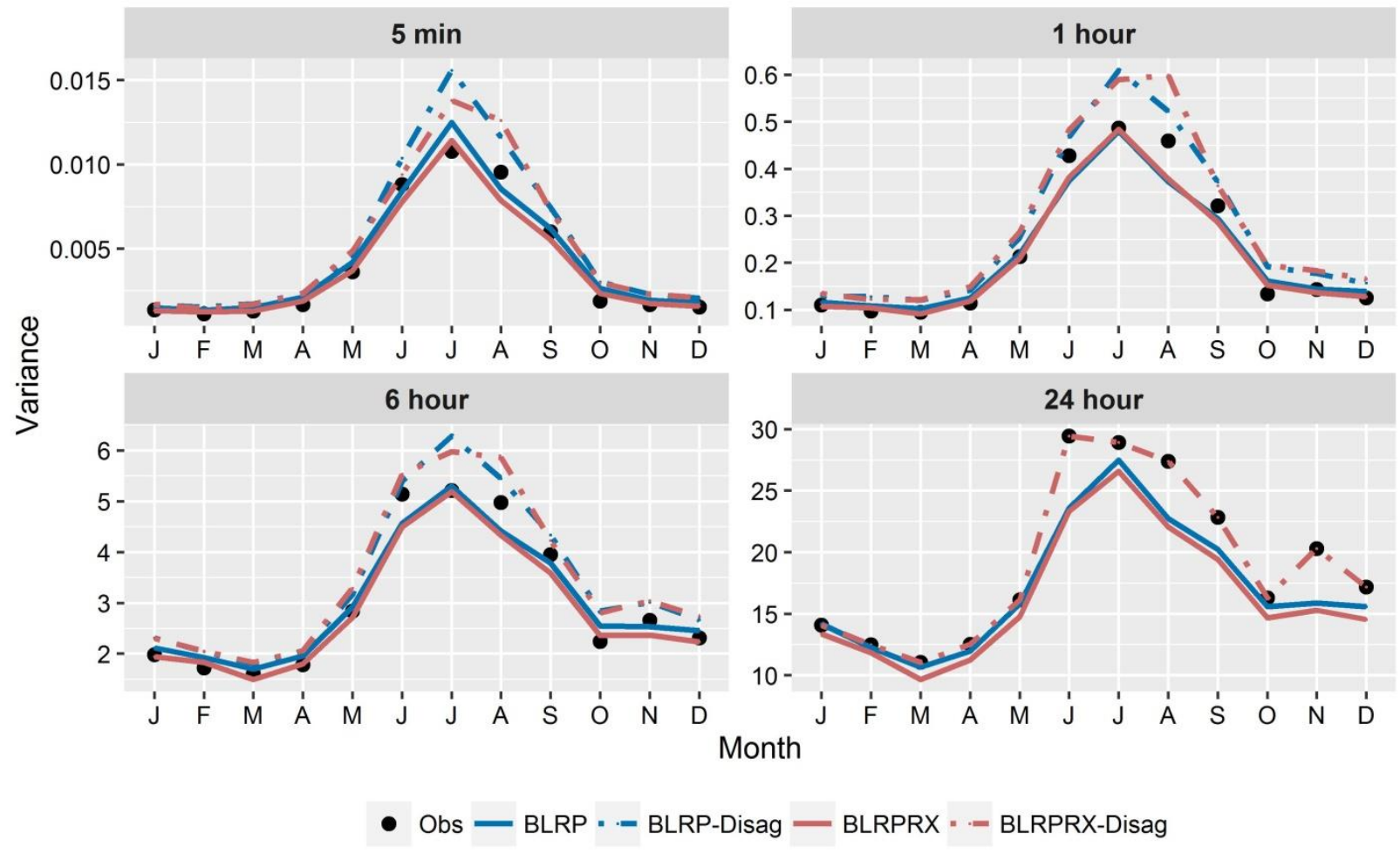

Fig. 4. Comparison of variance of the historical and synthetic data, by month. 

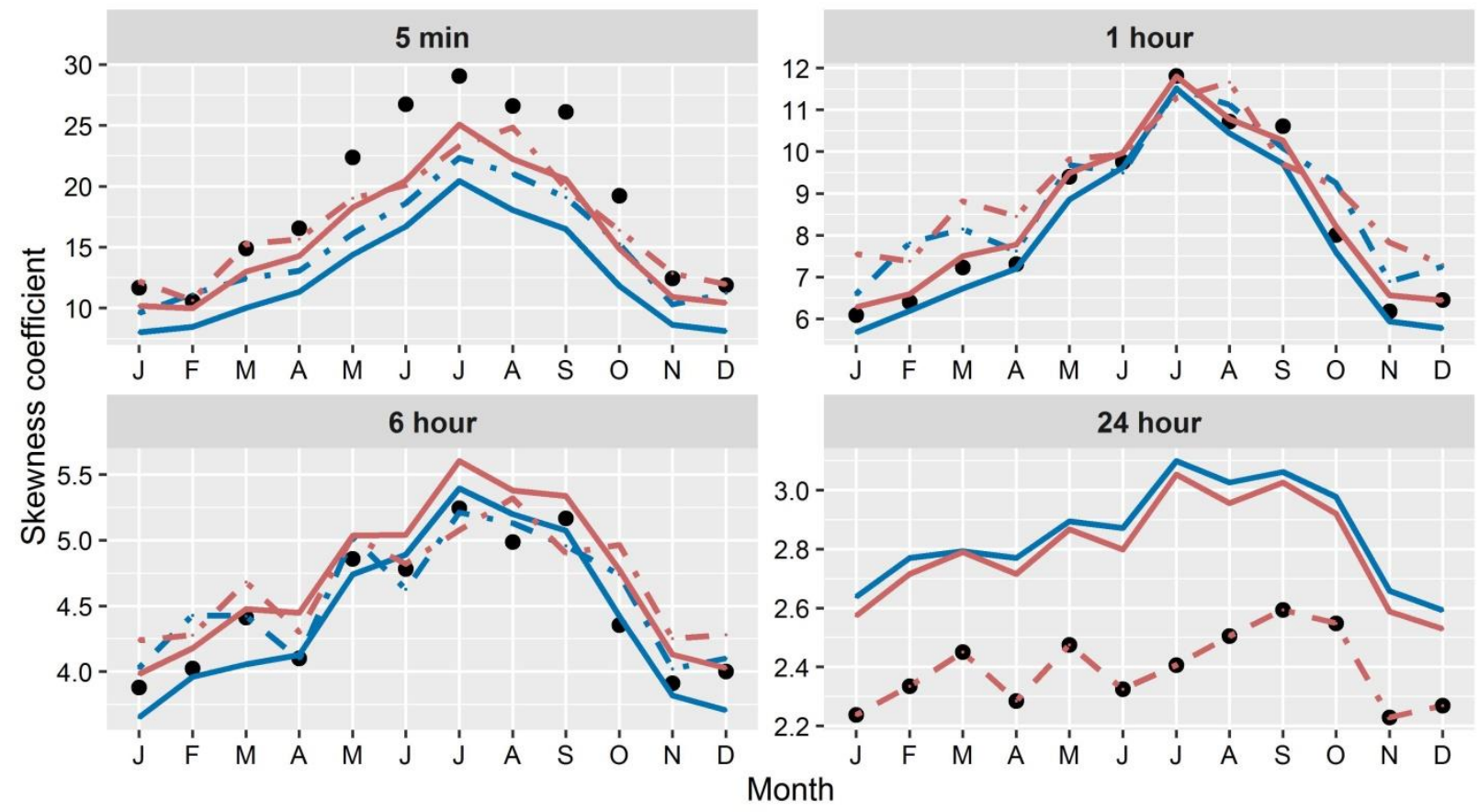

- Obs - BLRP . - BLRP-Disag - BLRPRX - - BLRPRX-Disag

Fig. 5. Comparison of coefficient of skewness of the historical and synthetic data, by month. 


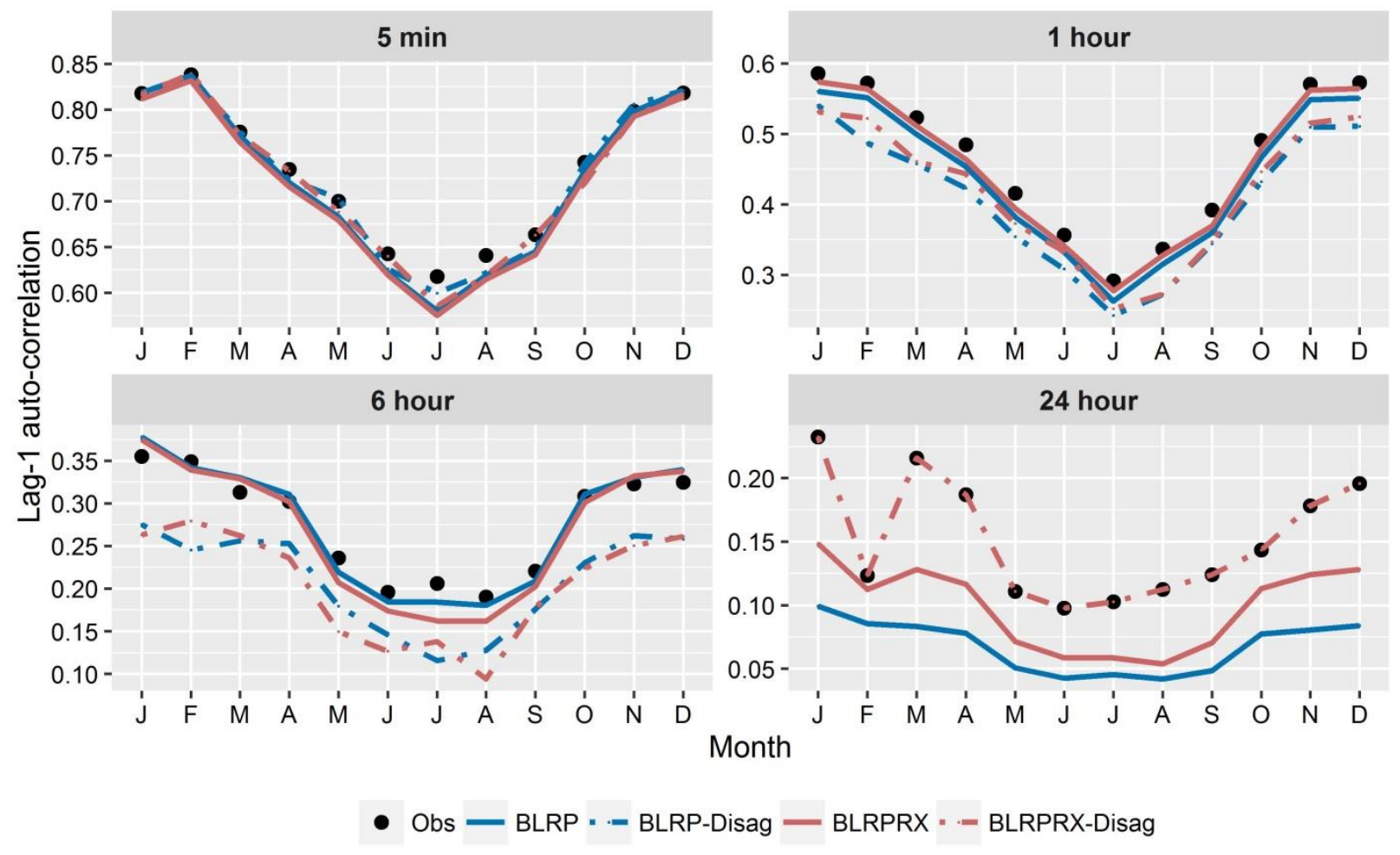

Fig. 6. Comparison of Lag-1 autocorrelation coefficient of the historical and synthetic data, by month.

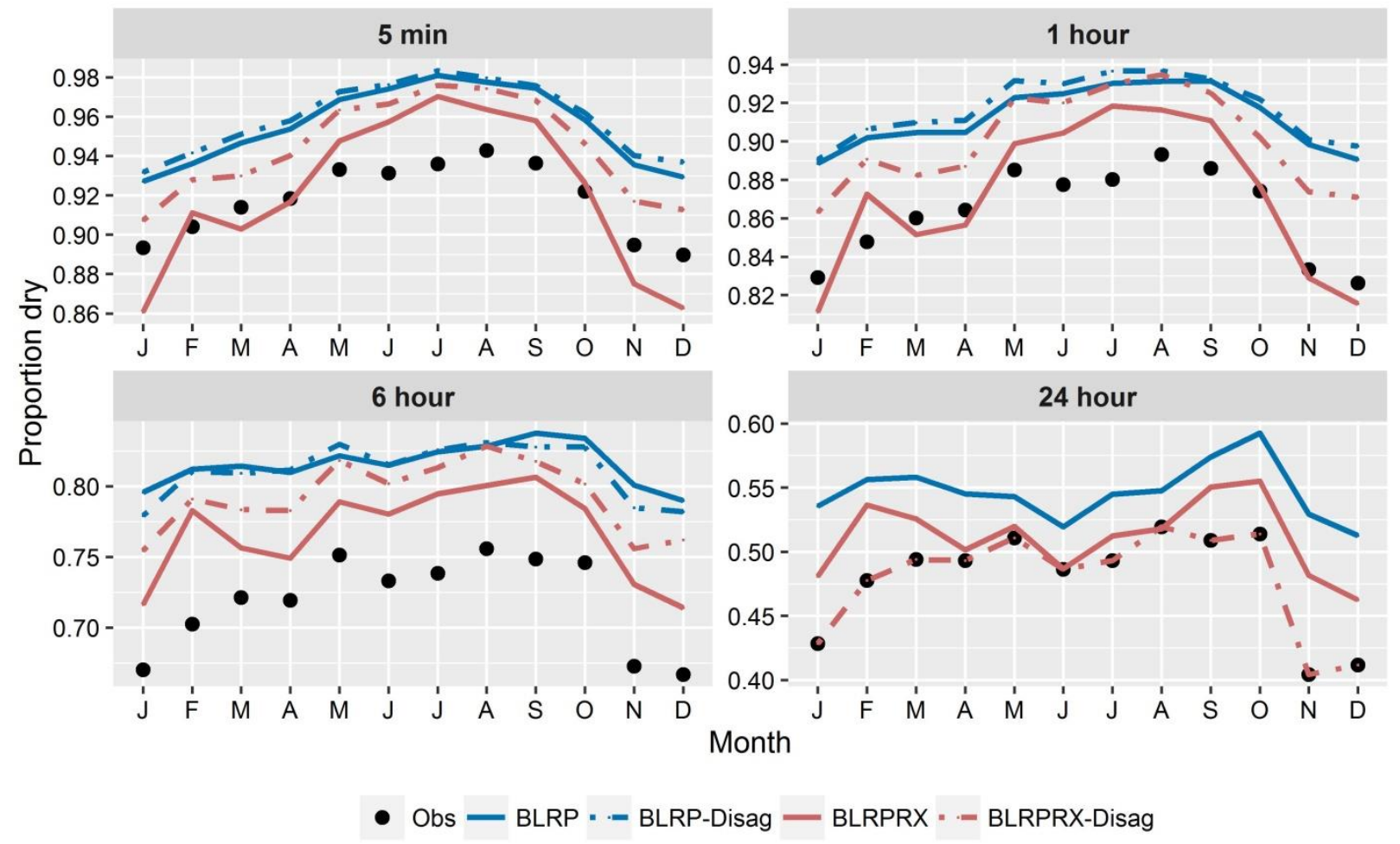

Fig. 7. Comparison of proportion of dry intervals of the historical and synthetic data, by month. 
Additionally, a series of rainfall depth conditional statistics are examined to assess the performance of the rainfall models as well as the disaggregation scheme. Specifically, the mean, variance and skewness coefficient of non-zero rainfall depths of simulated, disaggregated and historical data were obtained and depicted in Fig. 8 to Fig. 10. It should be mentioned that none of these properties were included in the fitting procedure and subsequently, their explicit preservation is not ensured by the models. As we observe both models tend to overestimate the mean of non-zero depths of summer months at all time scales of interest, while it is clear that the BLRPRx model performs better. The disaggregated series are in close agreement with the simulated without disaggregation, and hence we can say that no bias is introduced in that property. In terms of variance of nonzero values, simulated series from both models agree well with the historical series, while the BLRPRX model outperforms the original model. As in the variance of full series, the disaggregation creates a slight positive bias, especially in the 5-min time scale of summer months for which the variance is considerable high. Finally, Fig. 10 displays the skewness coefficient of non-zero values. As it is shown, both rainfall models considerably underestimate the conditional skewness at fine time scales (i.e., 5-minute and 1-hour), while significant improvement is achieved after applying the disaggregation model's adjustment procedure to the data generated by the BLRPRX model. 

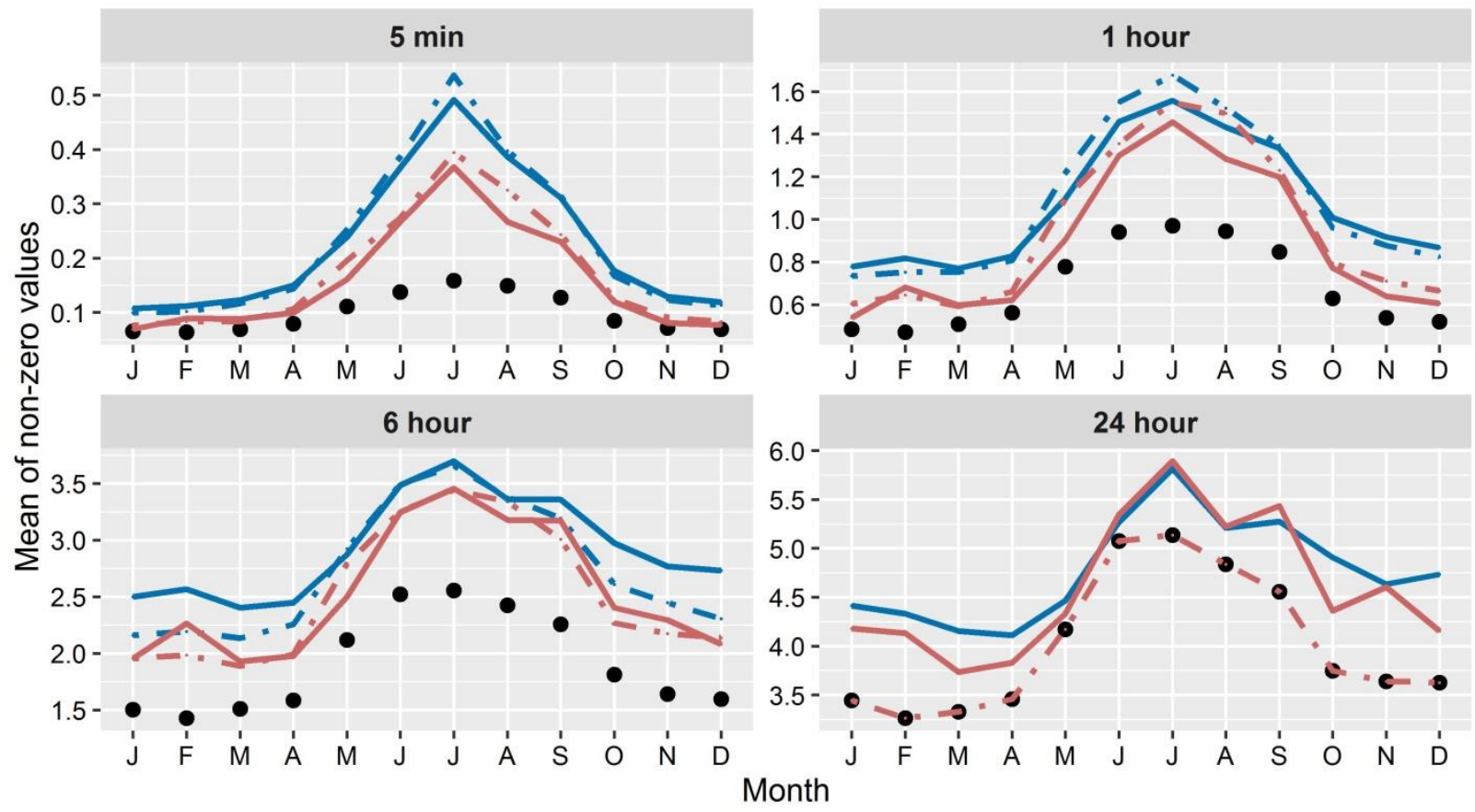

- Obs — BLRP - - BLRP-Disag - BLRPRX - - BLRPRX-Disag

Fig. 8. Comparison of mean of non-zero depths of the historical and synthetic data, by month. 

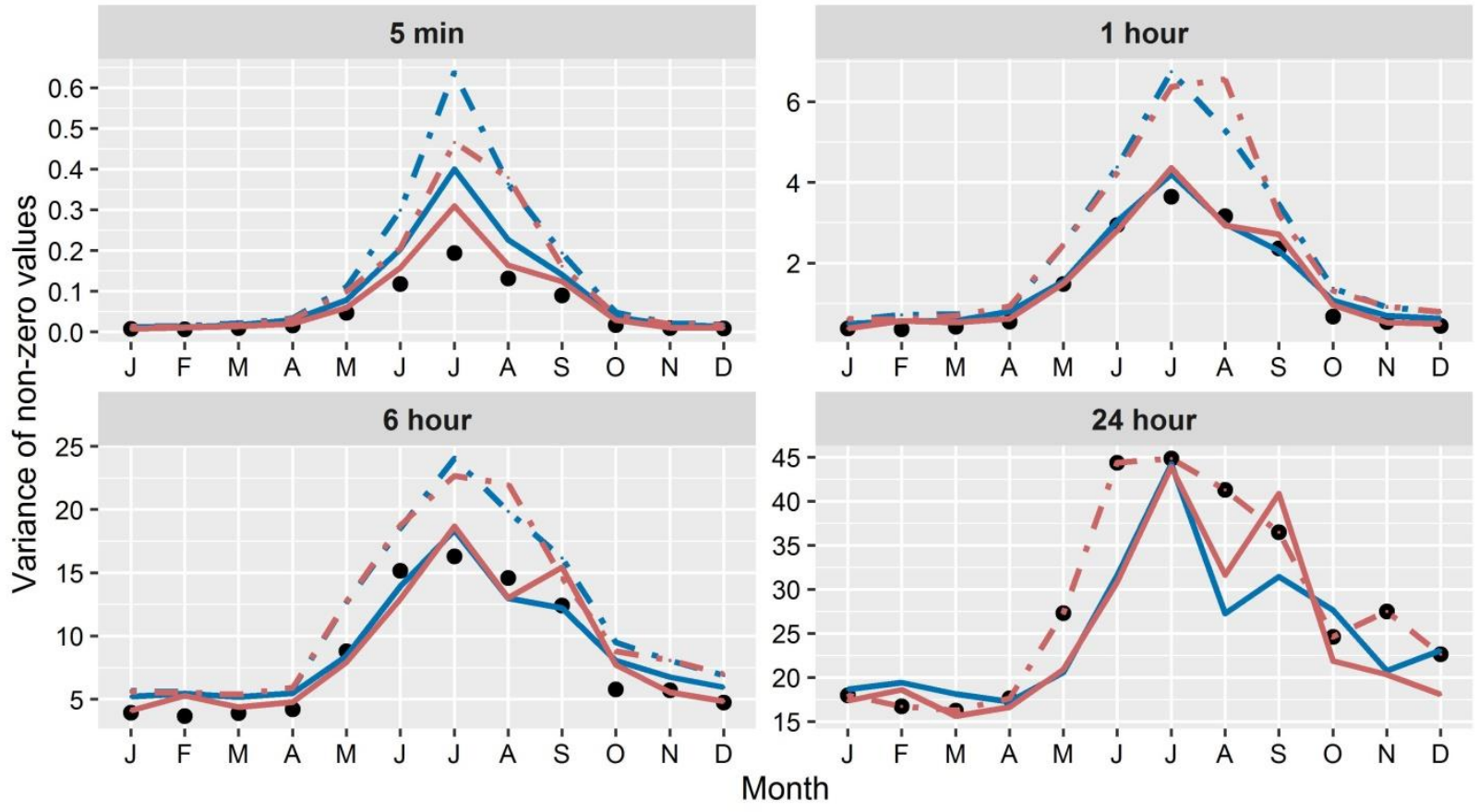

- Obs - BLRP . - BLRP-Disag — BLRPRX =- BLRPRX-Disag

Fig. 9. Comparison of variance of non-zero depths of the historical and synthetic data, by month. 


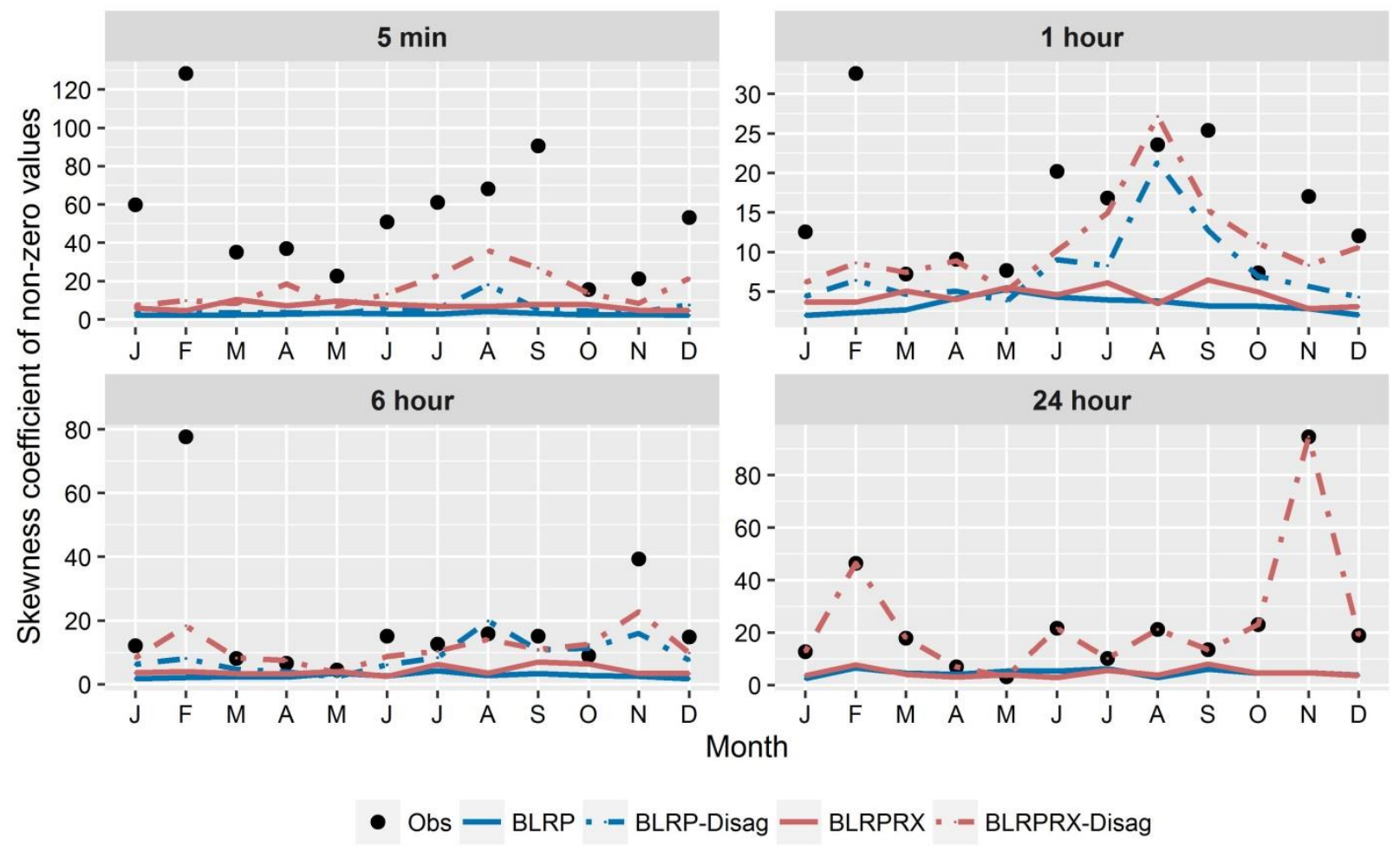

Fig. 10. Comparison of coefficient of skewness of non-zero depths of the historical and synthetic data, by month.

Finally, the performance of the disaggregation scheme in reproducing the distribution of maximum rainfall depths is examined. Specifically, data records from July were chosen due to the fact that this is the month with the highest variability and coefficient of skewness. The annual maximum rainfall depths of the historical, simulated and disaggregated series were plotted on Gumbel probability plots which are presented in Fig. 11. As it is shown, the synthetic data, generated via sequential simulation from RPBL and BLRPR $\mathrm{X}$ models, underestimate the extremes of the historical series, despite the fact that the latter model shows a better performance. In this type of simulation, the performance of the two models may be improved by incorporating a longer-tail distribution, such as gamma or Weibull, for cell intensities. On the contrary, the rainfall maxima obtained by 
disaggregation are in close agreement with the historical ones, while the new model with dependent cell intensity-duration outperforms the original one, for all time scales. Subsequently, the information contained in the daily totals and the positive bias introduced by the adjusting procedure account for the improvement brought about by disaggregation as opposed to mere simulation.

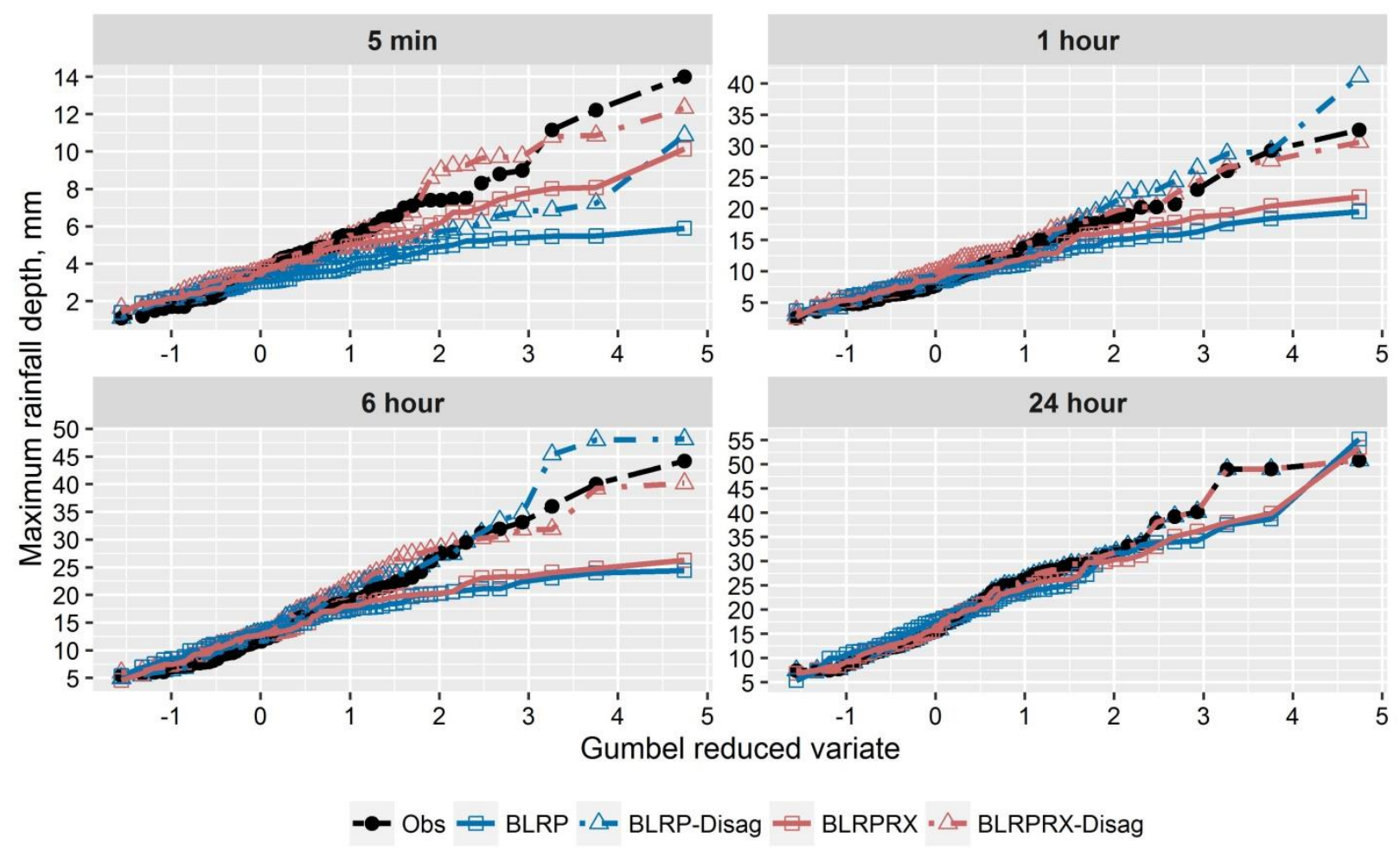

Fig. 11. Comparison of empirical distribution of maximum rainfall depths of the historical and synthetic data for July. 


\section{Conclusions and future directions}

Long rainfall time series at fine scales are required for many applications within the field of water resources and environmental management. However, such data are often limited in the real world. In order to cope with this problem, disaggregation techniques can be deployed to enhance the available data records with possible, statistically consistent, realizations of rainfall events that aggregate up to the field data collected at coarser scales.

This study extends a well-established disaggregation scheme, initially developed for the generation of hourly rainfall depths, to generate sub-hourly data down to the 1-minute time scale. Further to that and in order to enable the adequate reproduction of the high variability that rainfall exhibits at super-fine time scales, the recently developed BartlettLewis model that assumes dependence between cell intensity and duration was introduced in the scheme. This model is combined with proven transformations in order to adjust the generated rainfall depths so as to be consistent with the given daily data. To improve model performance and reduce the bias in the statistics that are not explicitly preserved by the process, a repetition scheme is incorporated in the process. In the framework of the present work, an R package, named HyetosMinute, which implements the extended disaggregation scheme as well as the rainfall model was developed.

As a test case of the proposed extension, the disaggregation of a daily rainfall series (from Bochum, Germany) into 5-minute depths was studied. Further to the new model with dependent intensity and duration (random with 6 parameters), as rainfall generator we also use the original Bartlett-Lewis model (non-random with 5 parameters) to enable comparisons. The results indicate that the statistical characteristics of disaggregated data, from both models, are in close agreement with those of observed data, while the model 
with randomised intensity parameter achieves a better performance with respect to skewness, proportion dry and rainfall extremes.

As a continuation of this work, our research will now focus on a series of challenging issues. Firstly, further assessment and validation of the proposed disaggregation methodology using rainfall datasets from different climatic conditions. The application of the disaggregation methodology without reference to data of the lower time scale should also be examined. This case corresponds to a fully operational scenario where the statistical properties of the target time scale are not introduced in the fitting procedure of the Bartlett-Lewis model. In the same framework, preliminary analysis showed that the performance of our disaggregation methodology is closely associated with the adequacy of the fit of the rainfall model to the observed statistics as well as with the properties that are included in the fitting procedure. This motivates a detailed sensitivity analysis of the disaggregation model to capture the stochastic structure implied by the rainfall model. Furthermore, the use of other types of distributions for cell intensities, e.g. gamma, Weibull and Pareto distribution, should also be considered in order to reveal the potential improvement in statistical characteristics such as skewness and extreme value performance. The comparison of the proposed methodology against other sub-hourly disaggregation methods with different theoretical backgrounds, such as multifractal modelling, cascade and micro-cascade techniques, is of particular importance to further investigate the advantages and disadvantages of the method. Finally, various extensions of the HyetosMinute package are already under development. These concern the incorporation of the new variants of the Bartlett-Lewis clustering mechanism, including the 
variants that imply a third level of temporal structure, as well as greater flexibility in the choice of distribution for cell intensities.

\section{Acknowledgements}

The authors gratefully acknowledge the Emschergenossenschaft and Lippeverband water companies in the North Rhine Westphalia region of Germany for the use of the Bochum rainfall data set. We would like to thank the two anonymous reviewers for their useful and constructive comments that improve the paper. All analyses were performed in the R programming environment (R Core Team, 2014), while the graphs in Section 4 were constructed using the ggplot2 package (Wickham, 2009).

\section{References}

Abdellatif, M., Atherton, W., Alkhaddar, R., 2013. Application of the stochastic model for temporal rainfall disaggregation for hydrological studies in north western England. J. Hydroinformatics 15, 555. doi:10.2166/hydro.2012.090

Anis, M.R., Rode, M., 2015. A new magnitude category disaggregation approach for temporal high-resolution rainfall intensities. Hydrol. Process. 29, 1119-1128. doi:10.1002/hyp.10227

Bennett, S.J., Bishop, T.F.A., Vervoort, R.W., 2013. Using SWAP to quantify space and time related uncertainty in deep drainage model estimates: A case study from northern NSW, Australia. Agric. Water Manag. 130, 142-153. doi:10.1016/j.agwat.2013.08.020

Bo, Z., Islam, S., Eltahir, E.A.B., 1994. Aggregation-disaggregation properties of a stochastic rainfall model, Water Resources Research. doi:10.1029/94WR02026

Burton, A., Kilsby, C.G., Fowler, H.J., Cowpertwait, P.S.P., O’Connell, P.E., 2008. RainSim: A 
spatial-temporal stochastic rainfall modelling system. Environ. Model. Softw. 23, 1356-1369. doi:10.1016/j.envsoft.2008.04.003

Cameron, D., Beven, K., Tawn, J., 2000. An evaluation of three stochastic rainfall models. J. Hydrol. 228, 130-149. doi:10.1016/S0022-1694(00)00143-8

Chandler, R., Lourmas, G., Jesus, J., 2010. Momfit: software for moment-based fitting of single-site stochastic rainfall models. Department of Statistical Science, University College London. Available at http://www.ucl.ac.uk/ ucakarc/work/momfit.html.

Cowpertwait, P., Isham, V., Onof, C., 2007. Point process models of rainfall: developments for fine-scale structure. Proc. R. Soc. a-Mathematical Phys. Eng. Sci. 463, 2569-2587. doi:DOI 10.1098/rspa.2007.1889

Cowpertwait, P.S.P., 2010. A spatial-temporal point process model with a continuous distribution of storm types. Water Resour. Res. 46. doi:10.1029/2010WR009728

Cowpertwait, P.S.P., 2004. Mixed rectangular pulses models of rainfall. Hydrol. Earth Syst. Sci. 8, 993-1000. doi:10.5194/hess-8-993-2004

Cowpertwait, P.S.P., 2002. A space-time Neyman-Scott model of rainfall: Empirical analysis of extremes. Water Resour. Res. 38, 1-14. doi:10.1029/2001WR000709

Cowpertwait, P.S.P., 1998. A Poisson-cluster model of rainfall: some high-order moments and extreme values. Proc. R. Soc. London A Math. Phys. Eng. Sci. 454, 885-898.

Cowpertwait, P.S.P., 1991. Further developments of the neyman-scott clustered point process for modeling rainfall. Water Resour. Res. 27, 1431-1438. doi:10.1029/91WR00479

Cowpertwait, P.S.P., Lockie, T., Davies, M.D., 2006. A stochastic spatial-temporal disaggregation model for rainfall. J. Hydrol. New Zeal. 45, 1-12. 
Cowpertwait, P.S.P., O'Connell, P.E., Metcalfe, a. V., Mawdsley, J. a., 1996a. Stochastic point process modelling of rainfall. I. Single-site fitting and validation. J. Hydrol. 175, 17-46. doi:10.1016/S0022-1694(96)80004-7

Cowpertwait, P.S.P., O'Connell, P.E., Metcalfe, a. V., Mawdsley, J. a., 1996b. Stochastic point process modelling of rainfall. II. Regionalisation and disaggregation. J. Hydrol. 175, 4765. doi:10.1016/S0022-1694(96)80005-9

Cox, D.R., Isham, V., 1988. A Simple Spatial-Temporal Model of Rainfall. Proc. R. Soc. London A Math. Phys. Eng. Sci. 415, 317-328.

Debele, B., Srinivasan, R., Yves Parlange, J., 2007. Accuracy evaluation of weather data generation and disaggregation methods at finer timescales. Adv. Water Resour. 30, 1286-1300. doi:10.1016/j.advwatres.2006.11.009

Derzekos, C., Koutsoyiannis, D., Onof, C., 2005. A new randomised Poisson cluster model for rainfall in time, in: European Geosciences Union General Assembly 2005, Geophysical Research Abstracts, Vol. 7, Vienna, 07236, European Geosciences Union, 2005. doi:10.1007/s10546-004-5452-y

Efstratiadis, A., Dialynas, Y.G., Kozanis, S., Koutsoyiannis, D., 2014. A multivariate stochastic model for the generation of synthetic time series at multiple time scales reproducing long-term persistence. Environ. Model. Softw. 62, 139-152. doi:10.1016/j.envsoft.2014.08.017

Efstratiadis, A., Koutsoyiannis, D., 2002. An evolutionary annealing-simplex algorithm for global optimisation of water resource systems Parts of the presentation Troubles encountered 1-23.

Efstratiadis, A., Nalbantis, I., Koutsoyiannis, D., 2015. Hydrological modelling of temporally- 
varying catchments: facets of change and the value of information. Hydrol. Sci. J. 60, 1438-1461. doi:10.1080/02626667.2014.982123

Engida, A.N., Esteves, M., 2011. Characterization and disaggregation of daily rainfall in the Upper Blue Nile Basin in Ethiopia. J. Hydrol. 399, 226-234. doi:10.1016/j.jhydrol.2011.01.001

Entekhabi, D., Rodriguez-Iturbe, I., Eagleson, P.S., 1989. Probabilistic representation of the temporal rainfall process by a modified Neyman-Scott Rectangular Pulses Model: Parameter estimation and validation. Water Resour. Res. 25, 295-302. doi:10.1029/WR025i002p00295

Glasbey, C.A., Cooper, G., McGechan, M.B., 1995. Disaggregation of daily rainfall by conditional simulation from a point-process model. J. Hydrol. 165, 1-9. doi:10.1016/0022-1694(94)02598-6

Grygier, J.C., Stedinger, J.R., 1988. Condensed disaggregation procedures and conservation corrections for stochastic hydrology. Water Resour. Res. 24, 1574-1584. doi:10.1029/WR024i010p01574

Gyasi-agyei, Y., 2005. Stochastic disaggregation of daily rainfall into one-hour time scale 309, 178-190. doi:10.1016/j.jhydrol.2004.11.018

Gyasi-Agyei, Y., 1999. Identification of regional parameters of a stochastic model for rainfall disaggregation. J. Hydrol. 223, 148-163. doi:10.1016/S0022-1694(99)00114-6

Gyasi-Agyei, Y., Mahbub, S.M.P. Bin, 2007. A stochastic model for daily rainfall disaggregation into fine time scale for a large region. J. Hydrol. 347, 358-370. doi:10.1016/j.jhydrol.2007.09.047

Gyasi-Agyei, Y., Willgoose, G.R., 1997. A hybrid model for point rainfall modeling. Water 
Resour. Res. 33, 1699-1706. doi:10.1029/97WR01004

Hingray, B., Monbaron, E., Jarrar, I., Favre, A.C., Consuegra, D., Musy, A., 2002. Stochastic generation and disaggregation of hourly rainfall series for continuous hydrological modelling and flood control reservoir design. Water Sci. Technol. 45, 113-119.

Islam, S., Entekhabi, D., Bras, R.L., Rodriguez-Iturbe, I., 1990. Parameter estimation and sensitivity analysis for the modified Bartlett-Lewis rectangular pulses model of rainfall. J. Geophys. Res. Atmos. 95, 2093-2100. doi:10.1029/JD095iD03p02093

Jesus, J., Chandler, R.E., 2011. Estimating functions and the generalized method of moments. Interface Focus 1, 871-885. doi:10.1098/rsfs.2011.0057

Kaczmarska, J., Isham, V., Onof, C., 2014. Point process models for fine-resolution rainfall. Hydrol. Sci. J. 140806131733005. doi:10.1080/02626667.2014.925558

Khaliq, M.N., Cunnane, C., 1996. Modelling point rainfall occurrences with the modified Bartlett-Lewis rectangular pulses model. J. Hydrol. 180, 109-138. doi:10.1016/00221694(95)02894-3

Kim, D., Cho, H., Onof, C., Choi, M., 2016. Let-It-Rain: a web application for stochastic point rainfall generation at ungaged basins and its applicability in runoff and flood modeling. Stoch. Environ. Res. Risk Assess. doi:10.1007/s00477-016-1234-6

Kim, D., Kwon, H.-H., Lee, S.-O., Kim, S., 2014. Regionalization of the Modified Bartlett-Lewis rectangular pulse stochastic rainfall model across the Korean Peninsula. J. HydroEnvironment Res. 1-15. doi:10.1016/j.jher.2014.10.004

Kim, D., Olivera, F., Cho, H., Lee, S.O., 2013a. Effect of the inter-annual variability of rainfall statistics on stochastically generated rainfall time series: Part 2. Impact on watershed response variables. Stoch. Environ. Res. Risk Assess. 27, 1611-1619. 
doi:10.1007/s00477-013-0697-y

Kim, D., Olivera, F., Cho, H., Socolofsky, S.A., 2013b. Regionalization of the modified bartlettlewis rectangular pulse stochastic rainfall model. Terr. Atmos. Ocean. Sci. 24, 421-436. doi:10.3319/TA0.2012.11.12.01(Hy)

Kossieris, P., Efstratiadis, A., Koutsoyiannis, D., 2013. Coupling the strengths of optimization and simulation for calibrating Poisson cluster models, in: Facets of Uncertainty: 5th EGU Leonardo Conference - Hydrofractals 2013 - STAHY 2013, Kos Island, Greece, European Geosciences Union, International Association of Hydrological Sciences, International Union of Geodesy and Geophysics, 2013.

Kossieris, P., Efstratiadis, A., Tsoukalas, I., Koutsoyiannis, D., 2015. Assessing the performance of Bartlett-Lewis model on the simulation of Athens rainfall, in: European Geosciences Union General Assembly 2015, Geophysical Research Abstracts, Vol. 17, Vienna, EGU2015-8983, European Geosciences Union, 2015.

Koutsoyiannis, D., 2011. Hurst-Kolmogorov Dynamics and Uncertainty. JAWRA J. Am. Water Resour. Assoc. 47, 481-495. doi:10.1111/j.1752-1688.2011.00543.x

Koutsoyiannis, D., 2003a. Rainfall disaggregation methods: theory and applications, in: Proceedings, Workshop on Statistical and Mathematical Methods for Hydrological Analysis, Università Degli Studi Di Roma “La Sapienza, Rome, P. 1-23.

Koutsoyiannis, D., 2003b. Climate change, the Hurst phenomenon, and hydrological statistics. Hydrol. Sci. J. 48, 3-24. doi:10.1623/hysj.48.1.3.43481

Koutsoyiannis, D., 2001. Coupling stochastic models of different time scales. Water Resour. Res. 37, 379-392.

Koutsoyiannis, D., 2000. A generalized mathematical framework for stochastic simulation 
and forecast of hydrologic time series. Water Resour. Res. 36, 1519-1533. doi:10.1029/2000WR900044

Koutsoyiannis, D., 1994. A stochastic disaggregation method for design storm and flood synthesis. J. Hydrol. 156, 193-225. doi:10.1016/0022-1694(94)90078-7

Koutsoyiannis, D., Foufoula-Georgiou, E., 1993. A scaling model of a storm hyetograph. Water Resour. Res. 29, 2345-2361. doi:10.1029/93WR00395

Koutsoyiannis, D., Manetas, A., 1996. Simple disaggregation by accurate adjusting procedures. Water Resour. Res. 32, 2105-2117. doi:10.1029/96WR00488

Koutsoyiannis, D., Onof, C., 2001. Rainfall disaggregation using adjusting procedures on a Poisson cluster model. J. Hydrol. 246, 109-122. doi:10.1016/S0022-1694(01)00363-8

Koutsoyiannis, D., Onof, C., 2000. A computer program for temporal rainfall disaggregation using adjusting procedures (HYETOS), in: 25th General Assembly of the European Geophysical Society, Geophysical Research Abstracts, Vol. 2, Nice, European Geophysical Society, 2000.

Koutsoyiannis, D., Onof, C., Wheater, H.S., 2003. Multivariate rainfall disaggregation at a fine timescale. Water Resour. Res. 39, n/a-n/a. doi:10.1029/2002WR001600

Laloy, E., Bielders, C.L., 2009. Modelling intercrop management impact on runoff and erosion in a continuous maize cropping system: Part II. Model Pareto multi-objective calibration and long-term scenario analysis using disaggregated rainfall. Eur. J. Soil Sci. 60, 1022-1037. doi:10.1111/j.1365-2389.2009.01190.x

Nalbantis, I., Efstratiadis, A., Rozos, E., Kopsiafti, M., Koutsoyiannis, D., 2011. Holistic versus monomeric strategies for hydrological modelling of human-modified hydrosystems. Hydrol. Earth Syst. Sci. 15, 743-758. doi:10.5194/hess-15-743-2011 
Nelder, J.A., Mead, R., 1965. A Simplex Method for Function Minimization. Comput. J. 7, 308313. doi:10.1093/comjnl/7.4.308

Onof, C., Chandler, R.E., Kakou, A., Northrop, P., Wheater, H.S., Isham, V., 2000. Rainfall modelling using Poisson-cluster processes: a review of developments. Stoch. Environ. Res. Risk Assess. 14, 384-411. doi:10.1007/s004770000043

Onof, C., Townend, J., Kee, R., 2005. Comparison of two hourly to 5-min rainfall disaggregators. Atmos. Res. 77, 176-187. doi:10.1016/j.atmosres.2004.10.022

Onof, C., Wheater, H.S., 1994a. Improvements to the modelling of British rainfall using a modified Random Parameter Bartlett-Lewis Rectangular Pulse Model. J. Hydrol. 157, 177-195. doi:10.1016/0022-1694(94)90104-X

Onof, C., Wheater, H.S., 1994b. Improved fitting of the Bartlett-Lewis Rectangular Pulse Model for hourly rainfall. Hydrol. Sci. J. 39, 663-680. doi:10.1080/02626669409492786

Onof, C., Wheater, H.S., 1993. Modelling of \uppercase\{B\}ritish rainfall using a random parameter \uppercase $\{\mathrm{B}\}$ artlett-\uppercase $\{\mathrm{L}\}$ ewis rectangular pulse model. J. Hydrol. 149, 67-95. doi:10.1016/0022-1694(93)90100-N

Onof, C., Wheater, H.S., Isham, V., 1994. Note on the analytical expression of the inter-event time characteristics for Bartlett-Lewis type rainfall models. J. Hydrol. 157, 197-210. doi:10.1016/0022-1694(94)90105-8

Paschalis, A., Molnar, P., Fatichi, S., Burlando, P., 2014. On temporal stochastic modeling of precipitation, nesting models across scales. Adv. Water Resour. 63, 152-166. doi:10.1016/j.advwatres.2013.11.006

Paschalis, A., Molnar, P., Fatichi, S., Burlando, P., 2013. A stochastic model for high- 
resolution space-time precipitation simulation. Water Resour. Res. 49, 8400-8417. doi:10.1002/2013WR014437

Pui, A., Sharma, A., Mehrotra, R., Sivakumar, B., Jeremiah, E., 2012. A comparison of alternatives for daily to sub-daily rainfall disaggregation. J. Hydrol. 470-471, 138-157. doi:10.1016/j.jhydrol.2012.08.041

R Core Team, 2014. R: A Language and Environment for Statistical Computing. Compstat 2008-Proceedings Comput. Stat.

Rodriguez-Iturbe, I., Cox, D.R., Isham, V., 1988. A Point Process Model for Rainfall: Further Developments. Proc. R. Soc. London A Math. Phys. Eng. Sci. 417, 283-298.

Rodriguez-Iturbe, I., Cox, D.R., Isham, V., 1987. Some Models for Rainfall Based on Stochastic Point Processes. Proc. R. Soc. Lond. A. Math. Phys. Sci. 410, 269-288.

Rodríguez-Iturbe, I., de Power, B.F., Valdés, J.B., 1987. Rectangular pulses point process models for rainfall: Analysis of empirical data. J. Geophys. Res. 92, 9645. doi:10.1029/JD092iD08p09645

Rodriguez-Iturbe, I., Gupta, V.K., Waymire, E., 1984. Scale considerations in the modeling of temporal rainfall. Water Resour. Res. 20, 1611-1619. doi:10.1029/WR020i011p01611

Segond, M.-L., Neokleous, N., Makropoulos, C., Onof, C., Maksimovic, C., 2007. Simulation and spatio-temporal disaggregation of multi-site rainfall data for urban drainage applications. Hydrol. Sci. J. 52, 917-935. doi:10.1623/hysj.52.5.917

Segond, M.-L., Onof, C., Wheater, H.S., 2006. Spatial-temporal disaggregation of daily rainfall from a generalized linear model. J. Hydrol. 331, 674-689. doi:10.1016/j.jhydrol.2006.06.019

Sivakumar, B., Sharma, A., 2008. A cascade approach to continuous rainfall data generation 
at point locations. Stoch. Environ. Res. Risk Assess. 22, 451-459. doi:10.1007/s00477007-0145-y

Smithers, J.C., Pegram, G.G.S., Schulze, R.E., 2002. Design rainfall estimation in South Africa using Bartlett-Lewis rectangular pulse rainfall models. J. Hydrol. 258, 83-99. doi:10.1016/S0022-1694(01)00571-6

Stedinger, J.R., Pei, D., Cohn, T.A., 1985. A condensed disaggregation model for incorporating parameter uncertainty into monthly reservoir simulations. Water Resour. Res. 21, 665-675. doi:10.1029/WR021i005p00665

Stedinger, J.R., Vogel, R.M., 1984. Disaggregation Procedures for Generating Serially Correlated Flow Vectors. Water Resour. Res. 20, 47-56. doi:10.1029/WR020i001p00047

Tsekouras, G., Koutsoyiannis, D., 2014. Stochastic analysis and simulation of hydrometeorological processes associated with wind and solar energy. Renew. Energy 63, 624-633. doi:10.1016/j.renene.2013.10.018

Tsoukalas, I., Kossieris, P., Efstratiadis, A., Makropoulos, C., 2016. Surrogate-enhanced evolutionary annealing simplex algorithm for effective and efficient optimization of water resources problems on a budget. Environ. Model. Softw. 77, 122-142. doi:10.1016/j.envsoft.2015.12.008

Tsoukalas, I., Makropoulos, C., 2015. Multiobjective optimisation on a budget: Exploring surrogate modelling for robust multi-reservoir rules generation under hydrological $\begin{array}{llll}\text { uncertainty. } & \text { Environ. }\end{array}$ doi:http://dx.doi.org/10.1016/j.envsoft.2014.09.023

Valencia R., D., Schaake L., J., 1972. A disaggregation model for time series analysis and 
synthesis.

Valencia, D., Schaake, J.C., 1973. Disaggregation Processes in Stochastic Hydrology. Water Resour. Res. 9, 580-585. doi:10.1029/WR009i003p00580

Velghe, T., Troch, P.A., De Troch, F.P., Van de Velde, J., 1994. Evaluation of cluster-based rectangular pulses point process models for rainfall. Water Resour. Res. 30, 28472857. doi:10.1029/94WR01496

Verhoest, N., Troch, P.A., De Troch, F.P., 1997. On the applicability of Bartlett-Lewis rectangular pulses models in the modeling of design storms at a point. J. Hydrol. 202, 108-120. doi:10.1016/S0022-1694(97)00060-7

Verhoest, N.E.C., Vandenberghe, S., Cabus, P., Onof, C., Meca-Figueras, T., Jameleddine, S., 2010. Are stochastic point rainfall models able to preserve extreme flood statistics? Hydrol. Process. 24, 3439-3445. doi:10.1002/hyp.7867

Wasko, C., Pui, A., Sharma, A., Mehrotra, R., Jeremiah, E., 2015. Representing low-frequency variability in continuous rainfall simulations: A hierarchical random Bartlett Lewis continuous rainfall generation model. Water Resour. Res. 51, 9995-10007. doi:10.1002/2015WR017469

Wheater, H.S., Chandler, R.E., Onof, C.J., Isham, V.S., Bellone, E., Yang, C., Lekkas, D., Lourmas, G., Segond, M.-L., 2005. Spatial-temporal rainfall modelling for flood risk estimation. Stoch. Environ. Res. Risk Assess. 19, 403-416. doi:10.1007/s00477-005-0011-8 